\title{
A PIECEWISE LINEAR FINITE ELEMENT METHOD FOR THE BUCKLING AND THE VIBRATION PROBLEMS OF THIN PLATES
}

\author{
DAVID MORA AND RODOLFO RODRÍGUEZ
}

\begin{abstract}
The aim of this paper is to analyze a piecewise linear finite element method to approximate the buckling and the vibration problems of a thin plate. The method is based on a conforming discretization of a bending moment formulation for the Kirchhoff-Love model. The analysis restricts to simply connected polygonal clamped plates, not necessarily convex. The method is proved to converge with optimal order for both spectral problems, including an improved order for the eigenvalues. Numerical experiments are reported to assess its performance and to compare it with other low-order finite element methods.
\end{abstract}

\section{INTRODUCTION}

The analysis of finite element methods to solve plate eigenvalue problems has a long history. Let us mention among the oldest references the papers by Canuto [6], Ishihara [13, 14, Rannacher [18, and Mercier et al. [17, Section 7(b,d)]. While [18 deals with non-conforming methods for the biharmonic equation, all the other papers are based on different mixed formulations of the Kirchhoff model. These formulations turn out to be equivalent to the biharmonic equation when the solution is smooth enough (typically $H^{3}$ ). Therefore, in order to allow for such regularity to hold (see [12]), the plate is assumed to be convex in these references.

One of the most well-known mixed methods to deal with the biharmonic equation is the method introduced by Ciarlet and Raviart 8 . This was thoroughly studied by many authors (see, for instance, [5, 20, [9, Section 3(a)], 4, Section 4(a)], 11, Section III.3], 10, 2]). The method was applied to the plate vibration problem in [6] and [17, Section 7(b)], where it was proved to converge for finite elements of degree $k \geq 2$. A formulation of the eigenvalue problem for the Stokes equation, which turns out to be equivalent to a plate buckling problem, is also analyzed in [17. Section $7(\mathrm{~d})$ ], where it is proved to converge for degree $k \geq 2$, as well. Although there is numerical evidence of optimal order convergence for piecewise linear elements applied to the vibration an the buckling plate problems (see in particular Section 5 below), to the best of our knowledge this has not been proved.

Received by the editor November 23, 2007 and, in revised form, September 3, 2008.

2000 Mathematics Subject Classification. Primary 65N25, 74K10, 65N30.

Key words and phrases. Buckling, Kirchhoff plates, spectral problems, low-order finite elements.

The first author was supported by a CONICYT fellowship (Chile).

The second author was partially supported by FONDAP and BASAL projects CMM, Universidad de Chile (Chile). 
Another classical mixed method to deal with Kirchhoff plates was introduced by Miyoshi in [16] for load problems. This method is based on piecewise linear elements and was extended by Ishihara to the vibration problem in [13] and to the buckling problem in [14. The method was proved to converge with a suboptimal order $\mathcal{O}\left(h^{1 / 2}\right)$, but only for meshes uniform in the interior of the domain. This hypothesis cannot be avoided. In fact, we report in Section 5 numerical experiments which show that this method converges to wrong results when used on particular regular non-uniform meshes.

Another low-order method was introduced much more recently by Amara et al. in [1] to deal with the load problem for a Kirchhoff-Love plate subject to arbitrary boundary conditions. This method is based on a standard discretization by loworder conforming elements of a bending moment formulation. In the present paper we adapt this approach to the buckling and the vibration problems. We restrict our analysis to simply connected polygonal clamped plates, not necessarily convex. In this case, all the equations are discretized by piecewise linear elements. We prove that the method leads to optimal orders of convergence for both the vibration and the buckling problems. Since the analysis of the former is very straightforward, we describe in detail only the latter and summarize the results for the former.

The outline of the paper is as follows: We introduce in Section 2 both eigenvalue problems. We recall the mixed formulation in terms of bending moments and a third equivalent formulation considered in [1, which allows using standard finite elements for its discretization. In Section 3 we develop the numerical analysis of the buckling problem. With this aim, we introduce a linear operator whose spectrum is related to the solution of the buckling problem. A spectral characterization is given and additional regularity results are proved. Then, the finite element method is introduced, and it is proved that it leads to optimal order approximation of the eigenfunctions. We end this section by proving that an improved order of convergence holds for the approximation of the eigenvalues. The same steps are briefly presented in Section 4 for the vibration problem, emphasizing the differences between both analyses. In Section 5 we report some numerical tests which confirm the theoretical results. We also include in this section numerical experiments with lowest-order Ciarlet-Raviart and Ishihara methods. These experiments show that the Ciarlet-Raviart method seems to converge with optimal order. The reported experiments also show that the Ishihara method fails when used on regular nonuniform meshes. We summarize some conclusions in Section 6. Finally, we give the matrix form of the discrete buckling problem in the Appendix, which allows us to prove a spectral characterization of this generalized eigenvalue problem.

\section{Problem statement}

Let $\Omega \subset \mathbb{R}^{2}$ be a polygonal bounded simply connected domain occupied by the mean surface of a plate, clamped on its whole boundary $\Gamma$. The plate is assumed to be homogeneous, isotropic, linearly elastic, and sufficiently thin as to be modeled by Kirchhoff-Love equations. We denote by $u$ the transverse displacement of the mean surface of the plate.

The plate vibration problem reads as follows:

Find $(\lambda, u) \in \mathbb{R} \times H^{2}(\Omega), u \neq 0$, such that

$$
\begin{cases}\Delta^{2} u=\lambda u & \text { in } \Omega, \\ u=\partial_{n} u=0 & \text { on } \Gamma,\end{cases}
$$


where $\lambda=\omega^{2}$, with $\omega>0$ being the vibration frequency, and $\partial_{n}$ denotes the normal derivative. To simplify the notation we have taken the Young modulus and the density of the plate, both equal to 1.

On the other hand, when the plate is subjected to a plane stress tensor field $\boldsymbol{\eta}: \Omega \rightarrow \mathbb{R}^{2 \times 2}$, the corresponding linear buckling problem reads as follows:

Find $(\lambda, u) \in \mathbb{R} \times H^{2}(\Omega), u \neq 0$, such that

$$
\begin{cases}\Delta^{2} u=-\lambda\left(\boldsymbol{\eta}: D^{2} u\right) & \text { in } \Omega, \\ u=\partial_{n} u=0 & \text { on } \Gamma,\end{cases}
$$

where in this case $\lambda$ is the critical load and $D^{2} u:=\left(\partial_{i j} u\right)_{1 \leq i, j \leq 2}$ denotes the Hessian matrix of $u$. The applied stress tensor field is assumed to satisfy the equilibrium equations:

$$
\begin{aligned}
\boldsymbol{\eta}^{\mathrm{T}}=\boldsymbol{\eta} & \text { in } \Omega, \\
\operatorname{div} \boldsymbol{\eta}=0 & \text { in } \Omega .
\end{aligned}
$$

Moreover, $\boldsymbol{\eta}$ is assumed to be essentially bounded, namely,

$$
\boldsymbol{\eta} \in L^{\infty}(\Omega)^{2 \times 2}
$$

However, we do not need to assume $\boldsymbol{\eta}$ to be positive definite. Let us remark that, in practice, $\boldsymbol{\eta}$ is the stress distribution on the plate subjected to in-plane loads, which does not need to be positive definite (see, for instance, Test 3 in Section 5.3 below).

Here and thereafter we use the following notation for any $2 \times 2$ tensor field $\tau$, any $2 \mathrm{D}$ vector field $\boldsymbol{v}$, and any scalar field $v$ :

$$
\begin{aligned}
& \operatorname{div} \boldsymbol{v}:=\partial_{1} v_{1}+\partial_{2} v_{2}, \quad \operatorname{curl} \boldsymbol{v}:=\partial_{1} v_{2}-\partial_{2} v_{1}, \quad \operatorname{curl} v:=\left(\begin{array}{c}
\partial_{2} v \\
-\partial_{1} v
\end{array}\right), \\
& \operatorname{div} \boldsymbol{\tau}:=\left(\begin{array}{l}
\partial_{1} \tau_{11}+\partial_{2} \tau_{12} \\
\partial_{1} \tau_{21}+\partial_{2} \tau_{22}
\end{array}\right), \quad \text { Curl } \boldsymbol{v}:=\left(\begin{array}{ll}
\partial_{2} v_{1} & -\partial_{1} v_{1} \\
\partial_{2} v_{2} & -\partial_{1} v_{2}
\end{array}\right) .
\end{aligned}
$$

Moreover, we denote

$$
\mathbf{I}:=\left(\begin{array}{ll}
1 & 0 \\
0 & 1
\end{array}\right), \quad \mathbf{J}:=\left(\begin{array}{rr}
0 & 1 \\
-1 & 0
\end{array}\right) .
$$

To obtain a weak formulation of each of the two spectral problems above, we multiply the corresponding equation by $v \in H_{0}^{2}(\Omega)$ and integrate twice by parts in $\Omega$. Thus, for the vibration problem (2.1) we obtain:

Find $(\lambda, u) \in \mathbb{R} \times H_{0}^{2}(\Omega), u \neq 0$, such that

$$
\int_{\Omega} \Delta u \Delta v=\lambda \int_{\Omega} u v \quad \forall v \in H_{0}^{2}(\Omega) .
$$

For the linear buckling problem we do the same and use the following lemma, which is easily proved by integrating by parts.

Lemma 2.1. For all $u \in H^{2}(\Omega), v \in H_{0}^{1}(\Omega)$, and $\boldsymbol{\eta}$ satisfying (2.3) $-(2.5)$,

$$
\int_{\Omega}\left(\boldsymbol{\eta}: D^{2} u\right) v=-\int_{\Omega}(\boldsymbol{\eta} \nabla u) \cdot \nabla v .
$$

Thus, we obtain the following symmetric weak formulation of the buckling prob$\operatorname{lem}(2.2)$ : 
Find $(\lambda, u) \in \mathbb{R} \times H_{0}^{2}(\Omega), u \neq 0$, such that

$$
\int_{\Omega} \Delta u \Delta v=\lambda \int_{\Omega}(\boldsymbol{\eta} \nabla u) \cdot \nabla v \quad \forall v \in H_{0}^{2}(\Omega) .
$$

It is well known that the eigenvalues of problem (2.6) are real and positive. Whenever $\boldsymbol{\eta}$ is positive definite, it is immediate to prove that those of problem (2.7) are real and positive, too. In any case these eigenvalues are real (see Lemma 3.1 below).

2.1. Formulation of the spectral problems in terms of bending moments. We follow an approach introduced and analyzed in [1] to deal with the load problem for Kirchhoff plates, adapted to the spectral problems of the previous section. Since the adaptation to the buckling problem presents several additional difficulties which must be tackled, we will describe this case in more detail and only summarize the analogous results for the vibration problem.

Let us denote

$$
\mathcal{V}:=H_{0}^{1}(\Omega) \quad \text { and } \quad \mathcal{X}:=\left\{\boldsymbol{\tau} \in L^{2}(\Omega)^{2 \times 2}: \operatorname{div}(\operatorname{div} \boldsymbol{\tau}) \in L^{2}(\Omega)\right\} .
$$

It was proved in [1] that $\mathcal{X}$ endowed with the norm

$$
\|\boldsymbol{\tau}\|_{\mathcal{X}}:=\left[\|\boldsymbol{\tau}\|_{0, \Omega}^{2}+\|\operatorname{div}(\operatorname{div} \boldsymbol{\tau})\|_{0, \Omega}^{2}\right]^{1 / 2}
$$

is a Hilbert space and that $\mathcal{D}(\bar{\Omega})^{2 \times 2}$ is a dense subspace of $\mathcal{X}$. Moreover,

$$
\int_{\Omega} \operatorname{div}(\operatorname{div} \boldsymbol{\tau}) v=\int_{\Omega} \boldsymbol{\tau}: D^{2} v \quad \forall \boldsymbol{\tau} \in \mathcal{X}, \quad \forall v \in H_{0}^{2}(\Omega) .
$$

Problem (2.7) can be rewritten as follows:

Find $(\lambda, \boldsymbol{\sigma}, u) \in \mathbb{R} \times \mathcal{X} \times H_{0}^{2}(\Omega), u \neq 0$, such that

$$
\begin{cases}\boldsymbol{\sigma}=\mathrm{C}\left(D^{2} u\right) & \text { in } \Omega, \\ \operatorname{div}(\operatorname{div} \boldsymbol{\sigma})=-\lambda \boldsymbol{\eta}: D^{2} u & \text { in } \Omega .\end{cases}
$$

In the expression above, $\boldsymbol{\sigma}=\left(\sigma_{i j}\right)_{1 \leq i, j \leq 2}$ is the so-called stress tensor and $\mathrm{C}$ is the linear operator arising from Hooke's law:

$$
\mathrm{C}(\boldsymbol{\tau}):=(1-\nu) \boldsymbol{\tau}+\nu(\operatorname{tr} \boldsymbol{\tau}) \mathbf{I}, \quad \boldsymbol{\tau} \in \mathbb{R}^{2 \times 2},
$$

with $\nu \in\left(0, \frac{1}{2}\right)$ being the Poisson coefficient. Let us remark that $\boldsymbol{\sigma}$ is a symmetric tensor as a consequence of the symmetry of $D^{2} u$.

The equivalence between problems (2.7) and (2.9) is a straightforward consequence of (2.8) and the identity

$$
\int_{\Omega} \mathrm{C}\left(D^{2} u\right): D^{2} v=\int_{\Omega} \Delta u \Delta v \quad \forall u, v \in H_{0}^{2}(\Omega),
$$

which in its turn follows from the density of $\mathcal{D}(\Omega)$ in $H_{0}^{2}(\Omega)$ and integration by parts.

To obtain a weak formulation of problem (2.9) we proceed as in [1. First note that the operator $\mathrm{C}$ is invertible, its inverse being given by

$$
\mathrm{C}^{-1}(\boldsymbol{\tau})=\frac{1}{1-\nu} \boldsymbol{\tau}-\frac{\nu}{1-\nu^{2}}(\operatorname{tr} \boldsymbol{\tau}) \mathbf{I}, \quad \boldsymbol{\tau} \in \mathbb{R}^{2 \times 2}
$$

Next, consider the following closed subspace of $\mathcal{X}$ :

$$
\mathcal{X}^{0}:=\{\boldsymbol{\tau} \in \mathcal{X}: \operatorname{div}(\operatorname{div} \boldsymbol{\tau})=0\} .
$$


The first equation of problem (2.9) can be equivalently written $\mathrm{C}^{-1}(\boldsymbol{\sigma})=D^{2} u$. By testing this equation with $\boldsymbol{\tau} \in \mathcal{X}^{0}$ and using (2.8), we obtain

$$
\int_{\Omega} C^{-1}(\boldsymbol{\sigma}): \boldsymbol{\tau}=\int_{\Omega} D^{2} u: \boldsymbol{\tau}=\int_{\Omega} \operatorname{div}(\operatorname{div} \boldsymbol{\tau}) u=0 \quad \forall \boldsymbol{\tau} \in \mathcal{X}^{0} .
$$

On the other hand, taking traces in the first equation of (2.9), it follows that $u$ is the unique solution of the problem

$$
\begin{cases}\Delta u-\frac{1}{1+\nu} \operatorname{tr} \sigma=0 & \text { in } \Omega, \\ u=0 & \text { on } \Gamma .\end{cases}
$$

Moreover, let $\phi$ be the solution of the problem

$$
\begin{cases}\Delta \phi=-\lambda \boldsymbol{\eta}: D^{2} u & \text { in } \Omega \\ \phi=0 & \text { on } \Gamma\end{cases}
$$

and let

$$
\boldsymbol{\sigma}^{0}:=\boldsymbol{\sigma}-\phi \mathbf{I}
$$

Since $\operatorname{div}(\operatorname{div} \phi \mathbf{I})=\Delta \phi$, from the second equation in (2.9) and the first one in (2.12), we have that $\operatorname{div}\left(\operatorname{div} \boldsymbol{\sigma}^{0}\right)=0$ and, hence, $\boldsymbol{\sigma}^{0} \in \boldsymbol{\mathcal { X }}^{0}$.

Therefore, by testing problems (2.11) and (2.12) with functions in $\mathcal{V}$, substituting $\boldsymbol{\sigma}=\boldsymbol{\sigma}^{0}+\phi \mathbf{I}$ in (2.10) and (2.11), and using Lemma 2.1, we arrive at the following weak formulation of problem (2.9):

Find $\left(\lambda, \phi, \boldsymbol{\sigma}^{0}, u\right) \in \mathbb{R} \times \mathcal{V} \times \mathcal{X}^{0} \times \mathcal{V}, u \neq 0$, such that

$$
\begin{cases}\int_{\Omega} \nabla \phi \cdot \nabla v=-\lambda \int_{\Omega}(\boldsymbol{\eta} \nabla u) \cdot \nabla v & \forall v \in \mathcal{V}, \\ \int_{\Omega} \mathrm{C}^{-1}\left(\boldsymbol{\sigma}^{0}+\phi \mathbf{I}\right): \boldsymbol{\tau}=0 & \forall \boldsymbol{\tau} \in \mathcal{X}^{0}, \\ \int_{\Omega} \nabla u \cdot \nabla \gamma+\frac{1}{1+\nu} \int_{\Omega}\left(\operatorname{tr} \boldsymbol{\sigma}^{0}+2 \phi\right) \gamma=0 & \forall \gamma \in \mathcal{V} .\end{cases}
$$

The following lemma will be used to prove that this problem is actually equivalent to problem (2.7).

Lemma 2.2. Given $\chi \in L^{2}(\Omega)^{2 \times 2}$, there holds $\int_{\Omega} \boldsymbol{\chi}: \boldsymbol{\tau}=0$ for all $\boldsymbol{\tau} \in \mathcal{X}^{0}$ if and only if there exists $v \in H_{0}^{2}(\Omega)$ such that $\chi=D^{2} v$.

Proof. Let $\chi \in L^{2}(\Omega)^{2 \times 2}$ be such that $\int_{\Omega} \chi: \boldsymbol{\tau}=0$ for all $\boldsymbol{\tau} \in \boldsymbol{\mathcal { X }}^{0}$. Let $v \in H_{0}^{2}(\Omega)$ be the solution of the following problem:

$$
\int_{\Omega} D^{2} v: D^{2} w=\int_{\Omega} \chi: D^{2} w \quad \forall w \in H_{0}^{2}(\Omega) .
$$

Hence, $\boldsymbol{\chi}-D^{2} v \in \mathcal{X}^{0}$ and, consequently,

$$
\int_{\Omega} \chi:\left(\chi-D^{2} v\right)=0
$$

On the other hand, testing the problem above with $w=v$, we have that

$$
\int_{\Omega}\left(\chi-D^{2} v\right): D^{2} v=0
$$

Subtracting this equation from the previous one, we obtain

$$
\int_{\Omega}\left(\chi-D^{2} v\right):\left(\chi-D^{2} v\right)=0
$$


and, hence, $\chi=D^{2} v$. Since the converse is a direct consequence of the definition of $\mathcal{X}^{0}$, we conclude the proof.

Now we are in a position to prove that problems (2.13) and (2.7) are equivalent.

Proposition 2.3. $\left(\lambda, \phi, \sigma^{0}, u\right)$ is a solution of problem (2.13) if and only if $(\lambda, u)$ is a solution of problem (2.7) and $\boldsymbol{\sigma}:=\boldsymbol{\sigma}^{0}+\phi \mathbf{I}=\mathrm{C}\left(D^{2} u\right)$.

Proof. It has already been shown that problems (2.7) and (2.9) are equivalent. So, it is enough to prove the equivalence between problems (2.13) and (2.9).

Let $\left(\lambda, \phi, \boldsymbol{\sigma}^{0}, u\right)$ be a solution of problem (2.13). The first equation of this problem and Lemma 2.1 imply that $\phi$ satisfies (2.12). Therefore, since $\boldsymbol{\sigma}^{0} \in \mathcal{X}^{0}$, $\boldsymbol{\sigma}:=\boldsymbol{\sigma}^{0}+\phi \mathbf{I}$ satisfies the second equation of (2.9).

On the other hand, the second equation of (2.13) and Lemma 2.2 imply that there exists $v \in H_{0}^{2}(\Omega)$ such that $\mathrm{C}^{-1}\left(\boldsymbol{\sigma}^{0}+\phi \mathbf{I}\right)=D^{2} v$ or, equivalently, $\boldsymbol{\sigma}^{0}+\phi \mathbf{I}=\mathrm{C}\left(D^{2} v\right)$. By taking traces in this expression, we observe that $v$ is the unique solution of the following problem:

$$
\begin{cases}\Delta v=\frac{1}{1+\nu}\left(\operatorname{tr} \sigma^{0}+2 \phi\right) & \text { in } \Omega, \\ v=0 & \text { on } \Gamma,\end{cases}
$$

whose weak form coincides with the third equation of problem (2.13). Consequently, $v=u$. Therefore, $u \in H_{0}^{2}(\Omega)$ and $\boldsymbol{\sigma}=\boldsymbol{\sigma}^{0}+\phi \mathbf{I}=\mathrm{C}\left(D^{2} u\right)$, which allows us to conclude that $(\lambda, \boldsymbol{\sigma}, u)$ is a solution of problem (2.9).

The converse has already been proved when deducing (2.9), so we conclude the proof.

Remark 2.4. Although no symmetry constraint is explicitly imposed in problem (2.13) on $\boldsymbol{\sigma}^{0}$ (and hence on $\boldsymbol{\sigma}=\boldsymbol{\sigma}^{0}+\phi \mathbf{I}$ ), according to the theorem above $\boldsymbol{\sigma}=$ $\mathrm{C}\left(D^{2} u\right)$. Consequently, $\boldsymbol{\sigma}$ and a fortiori the term $\boldsymbol{\sigma}^{0}$ in the solution of problem (2.13) turn out to be symmetric, anyway.

Analogously, the vibration problem (2.1) can be rewritten as follows:

Find $(\lambda, \boldsymbol{\sigma}, u) \in \mathbb{R} \times \mathcal{X} \times H_{0}^{2}(\Omega), u \neq 0$, such that

$$
\begin{cases}\boldsymbol{\sigma}=\mathrm{C}\left(D^{2} u\right) & \text { in } \Omega \\ \operatorname{div}(\operatorname{div} \boldsymbol{\sigma})=\lambda u & \text { in } \Omega\end{cases}
$$

The same arguments used for the buckling problem lead to the following weak formulation of this problem:

Find $\left(\lambda, \phi, \boldsymbol{\sigma}^{0}, u\right) \in \mathbb{R} \times \mathcal{V} \times \mathcal{X}^{0} \times \mathcal{V}, u \neq 0$, such that

$$
\begin{cases}\int_{\Omega} \nabla \phi \cdot \nabla v=-\lambda \int_{\Omega} u v & \forall v \in \mathcal{V}, \\ \int_{\Omega} C^{-1}\left(\boldsymbol{\sigma}^{0}+\phi \mathbf{I}\right): \boldsymbol{\tau}=0 & \forall \boldsymbol{\tau} \in \mathcal{X}^{0}, \\ \int_{\Omega} \nabla u \cdot \nabla \gamma+\frac{1}{1+\nu} \int_{\Omega}\left(\operatorname{tr} \boldsymbol{\sigma}^{0}+2 \phi\right) \gamma=0 & \forall \gamma \in \mathcal{V} .\end{cases}
$$

Finally, the following equivalence result holds true:

Proposition 2.5. $\left(\lambda, \phi, \boldsymbol{\sigma}^{0}, u\right)$ is a solution of problem (2.14) if and only if $(\lambda, u)$ is a solution of problem (2.6) and $\boldsymbol{\sigma}:=\boldsymbol{\sigma}^{0}+\phi \mathbf{I}=\mathrm{C}\left(D^{2} u\right)$. 
2.2. Equivalent variational formulations. Our next step is to introduce new variational formulations of the buckling and the vibration spectral problems, which allow using standard finite elements for their discretization. With this purpose, we follow once more the arguments proposed in [1] to obtain a convenient decomposition of the space $\mathcal{X}^{0}$.

Consider the following space:

$$
\mathcal{H}:=\left\{\boldsymbol{\xi} \in H^{1}(\Omega)^{2}: \int_{\Omega} \xi_{1}=0, \int_{\Omega} \xi_{2}=0 \text { and } \int_{\Omega} \operatorname{div} \boldsymbol{\xi}=0\right\},
$$

endowed with the norm

$$
\|\boldsymbol{\xi}\|_{\mathcal{H}}:=\left(\left\|\partial_{2} \xi_{1}\right\|_{0, \Omega}^{2}+\frac{1}{2}\left\|\partial_{2} \xi_{2}-\partial_{1} \xi_{1}\right\|_{0, \Omega}^{2}+\left\|\partial_{1} \xi_{2}\right\|_{0, \Omega}^{2}\right)^{1 / 2}
$$

It is shown in [1] that $\|\cdot\|_{\mathcal{H}}$ and $\|\cdot\|_{1, \Omega}$ are equivalent norms in $\mathcal{H}$, as a consequence of Korn's inequality.

In the same reference, it is also shown that, for each symmetric $\boldsymbol{\tau} \in \mathcal{X}^{0}$, there exists a unique $\boldsymbol{\xi} \in \mathcal{H}$ such that

$$
\boldsymbol{\tau}=\operatorname{Curl} \boldsymbol{\xi}+\frac{1}{2}(\operatorname{div} \boldsymbol{\xi}) \mathbf{J} .
$$

Since by virtue of Remark 2.4 the term $\sigma^{0}$ in the solution of problem (2.13) turns out to be symmetric, then it can be accordingly written

$$
\boldsymbol{\sigma}^{0}=\operatorname{Curl} \boldsymbol{\psi}+\frac{1}{2}(\operatorname{div} \boldsymbol{\psi}) \mathbf{J},
$$

for a unique $\boldsymbol{\psi} \in \mathcal{H}$.

Remark 2.6. The simple-connectedness assumption on $\Omega$ is necessary for the representation (2.15) to hold true for all symmetric $\boldsymbol{\tau} \in \mathcal{X}^{0}$. This is tacitly assumed in the proofs of [1, Section 4.1].

We introduce the following continuous bilinear form in $\mathcal{H}$ :

$$
\begin{aligned}
A(\boldsymbol{\psi}, \boldsymbol{\xi}):= & \int_{\Omega} \mathrm{C}^{-1}\left(\operatorname{Curl} \boldsymbol{\psi}+\frac{1}{2}(\operatorname{div} \boldsymbol{\psi}) \mathbf{J}\right):\left(\operatorname{Curl} \boldsymbol{\xi}+\frac{1}{2}(\operatorname{div} \boldsymbol{\xi}) \mathbf{J}\right) \\
= & \frac{1}{1-\nu} \int_{\Omega}\left[\partial_{2} \psi_{1} \partial_{2} \xi_{1}+\partial_{1} \psi_{2} \partial_{1} \xi_{2}+\frac{1}{2}\left(\partial_{2} \psi_{2}-\partial_{1} \psi_{1}\right)\left(\partial_{2} \xi_{2}-\partial_{1} \xi_{1}\right)\right] \\
& -\frac{\nu}{1-\nu^{2}} \int_{\Omega} \operatorname{curl} \boldsymbol{\psi} \operatorname{curl} \boldsymbol{\xi}
\end{aligned}
$$

Straightforward calculus leads to

$$
A(\boldsymbol{\xi}, \boldsymbol{\xi})=\frac{1}{1+\nu}\|\boldsymbol{\xi}\|_{\mathcal{H}}^{2}+\frac{\nu}{1-\nu^{2}} \int_{\Omega}\left[\left(\partial_{2} \xi_{1}+\partial_{1} \xi_{2}\right)^{2}+\left(\partial_{2} \xi_{2}-\partial_{1} \xi_{1}\right)^{2}\right],
$$

which shows that $A(\boldsymbol{\xi}, \boldsymbol{\xi}) \geq \frac{1}{1+\nu}\|\boldsymbol{\xi}\|_{\mathcal{H}}^{2}$ and, consequently, $A(\cdot, \cdot)$ is $\mathcal{H}$-elliptic.

On the other hand, explicit computations lead to

$$
\int_{\Omega} \mathrm{C}^{-1}(\phi \mathbf{I}):\left(\operatorname{Curl} \boldsymbol{\xi}+\frac{1}{2}(\operatorname{div} \boldsymbol{\xi}) \mathbf{J}\right)=-\frac{1}{1+\nu} \int_{\Omega} \phi \operatorname{curl} \boldsymbol{\xi}
$$

and

$$
\operatorname{tr} \boldsymbol{\sigma}^{0}=\operatorname{tr}\left(\operatorname{Curl} \boldsymbol{\psi}+\frac{1}{2}(\operatorname{div} \boldsymbol{\psi}) \mathbf{J}\right)=-\operatorname{curl} \boldsymbol{\psi}
$$

Using all this in problem (2.13), we obtain the following new formulation of the buckling problem: 
Find $(\lambda, \phi, \boldsymbol{\psi}, u) \in \mathbb{R} \times \mathcal{V} \times \mathcal{H} \times \mathcal{V}, u \neq 0$, such that

$$
\begin{cases}\int_{\Omega} \nabla \phi \cdot \nabla v=-\lambda \int_{\Omega}(\boldsymbol{\eta} \nabla u) \cdot \nabla v & \forall v \in \mathcal{V}, \\ A(\boldsymbol{\psi}, \boldsymbol{\xi})-\frac{1}{1+\nu} \int_{\Omega} \phi \operatorname{curl} \boldsymbol{\xi}=0 & \forall \boldsymbol{\xi} \in \mathcal{H}, \\ \int_{\Omega} \nabla u \cdot \nabla \gamma+\frac{1}{1+\nu} \int_{\Omega}(-\operatorname{curl} \boldsymbol{\psi}+2 \phi) \gamma=0 & \forall \gamma \in \mathcal{V} .\end{cases}
$$

In what follows we show that problems (2.13) and (2.19) are equivalent.

Proposition 2.7. $(\lambda, \phi, \boldsymbol{\psi}, u)$ is a solution of problem (2.19) if and only if $\left(\lambda, \phi, \boldsymbol{\sigma}^{0}, u\right)$ is a solution of problem (2.13), with $\boldsymbol{\sigma}^{0}=\operatorname{Curl} \boldsymbol{\psi}+\frac{1}{2}(\operatorname{div} \boldsymbol{\psi}) \mathbf{J}$.

Proof. Let $\left(\lambda, \phi, \boldsymbol{\sigma}^{0}, u\right)$ be a solution of problem (2.13). Let $\boldsymbol{\psi} \in \mathcal{H}$ be such that $\boldsymbol{\sigma}^{0}=\operatorname{Curl} \boldsymbol{\psi}+\frac{1}{2}(\operatorname{div} \boldsymbol{\psi}) \mathbf{J}$. Given $\boldsymbol{\xi} \in \mathcal{H}$, let $\boldsymbol{\tau}:=\operatorname{Curl} \boldsymbol{\xi}+\frac{1}{2}(\operatorname{div} \boldsymbol{\xi}) \mathbf{J} \in \boldsymbol{\mathcal { X }}^{0}$. Then, the last two equations in (2.19) follow from the corresponding ones in (2.13) by using (2.16)-(2.18).

Conversely, let $(\lambda, \phi, \psi, u)$ be a solution of problem (2.19) and $\sigma^{0}:=$ Curl $\psi+$ $\frac{1}{2}(\operatorname{div} \psi) \mathbf{J} \in \mathcal{X}^{0}$. The third equation in (2.19) and (2.18) yield the third equation in (2.13). On the other hand, the second equation in (2.19), (2.16), and (2.17) yield the second equation in (2.13), but only for symmetric test functions $\tau=$ Curl $\boldsymbol{\xi}+\frac{1}{2}(\operatorname{div} \boldsymbol{\xi}) \mathbf{J} \in \mathcal{X}^{0}$. To end the proof we will show that this equation also holds true for skew-symmetric test functions. In fact, since $\boldsymbol{\sigma}^{0}$ is symmetric, $\mathrm{C}^{-1}\left(\boldsymbol{\sigma}^{0}\right)$ is symmetric too, and so is $\mathrm{C}^{-1}(\phi \mathbf{I})$ as well. Hence, for any skew-symmetric $\boldsymbol{\tau} \in \mathcal{X}^{0}$, there holds $\int_{\Omega} \mathrm{C}^{-1}\left(\boldsymbol{\sigma}^{0}+\phi \mathbf{I}\right): \boldsymbol{\tau}=0$, and we conclude the proof.

Analogously, the vibration problem (2.14) can be written as follows:

Find $(\lambda, \phi, \psi, u) \in \mathbb{R} \times \mathcal{V} \times \mathcal{H} \times \mathcal{V}, u \neq 0$, such that

$$
\begin{cases}\int_{\Omega} \nabla \phi \cdot \nabla v=-\lambda \int_{\Omega} u v & \forall v \in \mathcal{V}, \\ A(\boldsymbol{\psi}, \boldsymbol{\xi})-\frac{1}{1+\nu} \int_{\Omega} \phi \operatorname{curl} \boldsymbol{\xi}=0 & \forall \boldsymbol{\xi} \in \mathcal{H}, \\ \int_{\Omega} \nabla u \cdot \nabla \gamma+\frac{1}{1+\nu} \int_{\Omega}(-\operatorname{curl} \boldsymbol{\psi}+2 \phi) \gamma=0 & \forall \gamma \in \mathcal{V} .\end{cases}
$$

The following equivalence result also holds true:

Proposition 2.8. $(\lambda, \phi, \boldsymbol{\psi}, u)$ is a solution of problem (2.20) if and only if $\left(\lambda, \phi, \boldsymbol{\sigma}^{0}, u\right)$ is a solution of problem (2.14), with $\boldsymbol{\sigma}^{0}=\operatorname{Curl} \boldsymbol{\psi}+\frac{1}{2}(\operatorname{div} \boldsymbol{\psi}) \mathbf{J}$.

Remark 2.9. In both problems, (2.19) and (2.20), the eigenvalues cannot vanish. In fact, in both cases, if $\lambda=0$, then the first equation yields $\phi=0$, the second one and the $\mathcal{H}$-ellipticity of $A$ lead to $\boldsymbol{\psi}=0$, and, from the third one, $u=0$. Moreover, $\int_{\Omega}(\boldsymbol{\eta} \nabla u) \cdot \nabla u \neq 0$ in problem (2.19), despite the fact that $\boldsymbol{\eta}$ is not necessarily positive definite. This is a consequence of the equivalence between problems (2.19) and (2.7) (cf. Propositions 2.7 and 2.3). Indeed, in problem (2.7), $\int_{\Omega}(\boldsymbol{\eta} \nabla u) \cdot \nabla u=0$ implies $\Delta u=0$ and, hence, $u=0$.

Finally, to end this section, we introduce a more compact notation for the spectral problems (2.19) and (2.20). Let $\mathscr{A}:(\mathcal{V} \times \mathcal{H} \times \mathcal{V}) \times(\mathcal{V} \times \mathcal{H} \times \mathcal{V}) \rightarrow \mathbb{R}$, 
$\mathscr{B}: L^{2}(\Omega) \times L^{2}(\Omega) \rightarrow \mathbb{R}$, and $\mathscr{C}: H^{1}(\Omega) \times H^{1}(\Omega) \rightarrow \mathbb{R}$ be the continuous and symmetric bilinear forms respectively defined by

$$
\begin{aligned}
& \mathscr{A}((\phi, \boldsymbol{\psi}, u),(\gamma, \boldsymbol{\xi}, v)):=A(\boldsymbol{\psi}, \boldsymbol{\xi})+\int_{\Omega} \nabla \phi \cdot \nabla v+\int_{\Omega} \nabla u \cdot \nabla \gamma \\
&-\frac{1}{1+\nu}\left[\int_{\Omega} \phi \operatorname{curl} \boldsymbol{\xi}+\int_{\Omega} \gamma \operatorname{curl} \boldsymbol{\psi}\right]+\frac{2}{1+\nu} \int_{\Omega} \phi \gamma, \\
& \mathscr{B}(u, v):=\int_{\Omega} u v \\
& \mathscr{C}(u, v):=\int_{\Omega}(\boldsymbol{\eta} \nabla u) \cdot \nabla v .
\end{aligned}
$$

Using this notation, problems (2.19) and (2.20) can be respectively written as follows:

Find $(\lambda, \phi, \boldsymbol{\psi}, u) \in \mathbb{R} \times \mathcal{V} \times \mathcal{H} \times \mathcal{V}, u \neq 0$, such that

$$
\mathscr{A}((\phi, \boldsymbol{\psi}, u),(\gamma, \boldsymbol{\xi}, v))=-\lambda \mathscr{C}(u, v) \quad \forall(\gamma, \boldsymbol{\xi}, v) \in \mathcal{V} \times \mathcal{H} \times \mathcal{V} .
$$

Find $(\lambda, \phi, \boldsymbol{\psi}, u) \in \mathbb{R} \times \mathcal{V} \times \mathcal{H} \times \mathcal{V}, u \neq 0$, such that

$$
\mathscr{A}((\phi, \boldsymbol{\psi}, u),(\gamma, \boldsymbol{\xi}, v))=-\lambda \mathscr{B}(u, v) \quad \forall(\gamma, \boldsymbol{\xi}, v) \in \mathcal{V} \times \mathcal{H} \times \mathcal{V} .
$$

\section{NUMERICAL ANALYSIS OF THE BUCKLING PROBLEM}

Before introducing the numerical method, we define the linear operator corresponding to the source problem associated with the buckling spectral problem (2.21) and prove some properties that will be used for the subsequent convergence analysis. Consider the following source problem:

Given $f \in \mathcal{V}$, find $(\phi, \psi, u) \in \mathcal{V} \times \mathcal{H} \times \mathcal{V}$ such that

$$
\mathscr{A}((\phi, \boldsymbol{\psi}, u),(\gamma, \boldsymbol{\xi}, v))=-\mathscr{C}(f, v) \quad \forall(\gamma, \boldsymbol{\xi}, v) \in \mathcal{V} \times \mathcal{H} \times \mathcal{V}
$$

This problem is well posed. In fact, it can be decomposed into the following sequence of three well posed problems:

(1) Find $\phi \in \mathcal{V}$ such that

$$
\int_{\Omega} \nabla \phi \cdot \nabla v=-\int_{\Omega}(\boldsymbol{\eta} \nabla f) \cdot \nabla v \quad \forall v \in \mathcal{V} .
$$

(2) Find $\boldsymbol{\psi} \in \mathcal{H}$ such that

$$
A(\boldsymbol{\psi}, \boldsymbol{\xi})=G^{\phi}(\boldsymbol{\xi}):=\frac{1}{1+\nu} \int_{\Omega} \phi \operatorname{curl} \boldsymbol{\xi} \quad \forall \boldsymbol{\xi} \in \mathcal{H} .
$$

(3) Find $u \in \mathcal{V}$ such that

$$
\int_{\Omega} \nabla u \cdot \nabla \gamma=R^{\phi, \psi}(\gamma):=\frac{1}{1+\nu} \int_{\Omega}(\operatorname{curl} \boldsymbol{\psi}-2 \phi) \gamma \quad \forall \gamma \in \mathcal{V} .
$$

Let $T$ be the bounded linear operator defined by

$$
\begin{aligned}
T: \mathcal{V} & \rightarrow \mathcal{V}, \\
f & \mapsto u,
\end{aligned}
$$

with $(\phi, \boldsymbol{\psi}, u) \in \mathcal{V} \times \boldsymbol{H} \times \mathcal{V}$ being the solution of (3.1). Clearly $\lambda$ is an eigenvalue of problem (2.21) if and only if $\mu:=\frac{1}{\lambda}$ is a non-zero eigenvalue of $T$, with the same multiplicity and corresponding eigenfunctions $u$ (recall $\lambda \neq 0$; cf. Remark 2.9). 
The arguments used in the previous sections applied now to problem (3.1) allow us to show its equivalence with the following one:

Given $f \in \mathcal{V}$, find $u \in H_{0}^{2}(\Omega)$ such that

$$
\int_{\Omega} \Delta u \Delta v=\int_{\Omega}(\boldsymbol{\eta} \nabla f) \cdot \nabla v \quad \forall v \in H_{0}^{2}(\Omega) .
$$

More precisely, $u$ coincides in both problems and

$$
\boldsymbol{\sigma}:=\mathrm{C}\left(D^{2} u\right)=\operatorname{Curl} \boldsymbol{\psi}+\frac{1}{2}(\operatorname{div} \boldsymbol{\psi}) \mathbf{J}+\phi \mathbf{I} .
$$

As a consequence, we can prove the following spectral characterization:

Lemma 3.1. The spectrum of $T$ satisfies $\operatorname{sp}(T)=\{0\} \cup\left\{\mu_{n}: n \in \mathbb{N}\right\}$, where $\left\{\mu_{n}\right\}_{n \in \mathbb{N}}$ is a sequence of real eigenvalues which converges to 0 . The multiplicity of each non-zero eigenvalue is finite and its ascent is 1.

Proof. By virtue of the equivalence between problems (3.1) and (3.5), $T$ is also a bounded linear operator from $\mathcal{V}$ into $H_{0}^{2}(\Omega)$. Hence, because of the compact inclusion $H_{0}^{2}(\Omega) \hookrightarrow \mathcal{V}$ and the spectral characterization of compact operators, we have that $\operatorname{sp}(T)=\{0\} \cup\left\{\mu_{n}: n \in \mathbb{N}\right\}$, with $\left\{\mu_{n}\right\}_{n \in \mathbb{N}}$ a sequence of finite-multiplicity eigenvalues which converges to 0 .

Moreover, it is simple to prove by using (2.3) that $\left.T\right|_{H_{0}^{2}(\Omega)}: H_{0}^{2}(\Omega) \rightarrow H_{0}^{2}(\Omega)$ is self-adjoint with respect to the inner product $(u, v) \mapsto \int_{\Omega} \Delta u \Delta v$. Therefore, since $\operatorname{sp}(T)=\{0\} \cup \operatorname{sp}\left(\left.T\right|_{H_{0}^{2}(\Omega)}\right)$, we conclude that the non-zero eigenvalues of $T$ are real and have ascent 1 . Thus we end the proof.

Another conclusion of the equivalence between problems (3.1) and (3.5) is the following additional regularity result.

Lemma 3.2. There exist $s \in\left(\frac{1}{2}, 1\right]$ and $C>0$ such that, for all $f \in \mathcal{V}$, the solution $(\phi, \boldsymbol{\psi}, u)$ of problem (3.1) satisfies $u \in H^{2+s}(\Omega), \boldsymbol{\psi} \in H^{1+s}(\Omega)^{2}$, and

$$
\|\phi\|_{1, \Omega}+\|u\|_{2+s, \Omega}+\|\boldsymbol{\psi}\|_{1+s, \Omega} \leq C\|f\|_{1, \Omega} .
$$

Proof. The estimate for $\phi$ (which does not involve any additional regularity) follows directly from (3.2) and (2.5). The estimate for $u$ follows from the equivalence between problems (3.1) and (3.5) and the classical regularity result for the biharmonic problem with its right-hand side in $H^{-1}(\Omega)$ (cf. [12]).

To prove the estimate for $\psi$, we use the explicit expression (2.16) for $A$ to write

$$
A(\boldsymbol{\psi}, \boldsymbol{\xi})=\frac{1}{1-\nu} \int_{\Omega} \varepsilon(\tilde{\boldsymbol{\psi}}): \varepsilon(\tilde{\boldsymbol{\xi}})-\frac{\nu}{1-\nu^{2}} \int_{\Omega} \operatorname{div} \tilde{\boldsymbol{\psi}} \operatorname{div} \tilde{\boldsymbol{\xi}}
$$

with $\tilde{\boldsymbol{\psi}}=\left(\psi_{2},-\psi_{1}\right), \tilde{\boldsymbol{\xi}}=\left(\xi_{2},-\xi_{1}\right)$, and $\boldsymbol{\varepsilon}=\left(\varepsilon_{i j}\right)_{1 \leq i, j \leq 2}$ being the standard strain tensor defined by $\varepsilon_{i j}(\boldsymbol{v}):=\frac{1}{2}\left(\partial_{i} v_{j}+\partial_{j} v_{i}\right), 1 \leq i, j \leq 2$. By substituting (3.6) into (3.3) and integrating by parts the right-hand side, we find that $\tilde{\boldsymbol{\psi}}$ is the solution of an elasticity-like problem with Lamé coefficients $\tilde{\mu}:=\frac{1}{2(1-\nu)}$ and $\tilde{\lambda}:=-\frac{\nu}{1-\nu^{2}}$, source term $-\frac{1}{1+\nu} \nabla \phi \in L^{2}(\Omega)^{2}$, and traction free boundary conditions. Notice that $\tilde{\mu}>0$ and $\tilde{\lambda}+\tilde{\mu}=\frac{1}{2(1+\nu)}>0$, too. Moreover, since the source term is orthogonal to the set of rigid motions, because of the constraints in the definition of $\mathcal{H}$, the elasticity-like problem is well posed. Hence, from a classical regularity result for 
the elasticity equations (see, for instance, [19, Theorem 5.2]), there exists $s \in\left(\frac{1}{2}, 1\right]$ such that $\tilde{\psi}$ and a fortiori $\boldsymbol{\psi}$ satisfy

$$
\|\boldsymbol{\psi}\|_{1+s, \Omega} \leq C\|\nabla \phi\|_{0, \Omega} \leq C\|f\|_{1, \Omega} .
$$

Thus we conclude the proof.

Remark 3.3. The constant $s$ in the lemma above is the Sobolev regularity for the biharmonic equation with the right-hand side in $H^{-1}(\Omega)$ and homogeneous Dirichlet boundary conditions. In fact, for the linear elasticity equations with the right-hand side in $L^{2}(\Omega)^{2}$ and purely homogeneous Neumann conditions, the Sobolev regularity $s$ is the same one. This constant only depends on the domain $\Omega$. If $\Omega$ is convex, then $s=1$. Otherwise, the lemma holds for all $s<s_{0}$, where $s_{0} \in\left(\frac{1}{2}, 1\right)$ depends on the largest reentrant angle of $\Omega$ (see 12 for the precise equation determining $\left.s_{0}\right)$.

Remark 3.4. The lemma above does not fix any further regularity for $\phi$. Indeed, no additional regularity can be expected for arbitrary $f \in \mathcal{V}$. For instance, from (3.2), if $\boldsymbol{\eta}=\mathbf{I}$, then $\phi \equiv f$.

3.1. Finite element approximation. For the numerical approximation, we consider a regular family $\left\{\mathcal{T}_{h}\right\}_{h>0}$ of triangular meshes in $\bar{\Omega}$ and the standard piecewise linear continuous finite element space

$$
\mathcal{L}_{h}:=\left\{v_{h} \in \mathcal{C}(\bar{\Omega}):\left.v_{h}\right|_{T} \in \mathcal{P}_{1}(T) \quad \forall T \in \mathcal{T}_{h}\right\} .
$$

Let $\mathcal{V}_{h}$ and $\mathcal{H}_{h}$ be the finite-dimensional subspaces of $\mathcal{V}$ and $\mathcal{H}$, respectively defined by

$$
\begin{aligned}
\mathcal{V}_{h} & :=\mathcal{L}_{h} \cap \mathcal{V}=\left\{v_{h} \in \mathcal{L}_{h}: v_{h}=0 \text { on } \Gamma\right\} \\
\mathcal{H}_{h} & :=\mathcal{L}_{h}^{2} \cap \mathcal{H}=\left\{\boldsymbol{\xi}_{h} \in \mathcal{L}_{h}^{2}: \int_{\Omega} \xi_{h 1}=0, \int_{\Omega} \xi_{h 2}=0 \text { and } \int_{\Omega} \operatorname{div} \boldsymbol{\xi}_{h}=0\right\} .
\end{aligned}
$$

The discrete version of problem (2.21) reads as follows:

Find $\left(\lambda_{h}, \phi_{h}, \boldsymbol{\psi}_{h}, u_{h}\right) \in \mathbb{R} \times \mathcal{V}_{h} \times \mathcal{H}_{h} \times \mathcal{V}_{h}, u_{h} \neq 0$, such that

$$
\mathscr{A}\left(\left(\phi_{h}, \boldsymbol{\psi}_{h}, u_{h}\right),\left(\gamma_{h}, \boldsymbol{\xi}_{h}, v_{h}\right)\right)=-\lambda_{h} \mathscr{C}\left(u_{h}, v_{h}\right) \quad \forall\left(\gamma_{h}, \boldsymbol{\xi}_{h}, v_{h}\right) \in \mathcal{V}_{h} \times \mathcal{H}_{h} \times \mathcal{V}_{h} .
$$

Let $T_{h}$ be the bounded linear operator defined by

$$
\begin{aligned}
T_{h}: \mathcal{V} & \rightarrow \mathcal{V}, \\
f & \mapsto u_{h},
\end{aligned}
$$

with $\left(\phi_{h}, \boldsymbol{\psi}_{h}, u_{h}\right) \in \mathcal{V}_{h} \times \mathcal{H}_{h} \times \mathcal{V}_{h}$ being the solution of the discrete analog of problem (3.1):

$$
\mathscr{A}\left(\left(\phi_{h}, \boldsymbol{\psi}_{h}, u_{h}\right),\left(\gamma_{h}, \boldsymbol{\xi}_{h}, v_{h}\right)\right)=-\mathscr{C}\left(f, v_{h}\right) \quad \forall\left(\gamma_{h}, \boldsymbol{\xi}_{h}, v_{h}\right) \in \mathcal{V}_{h} \times \mathcal{H}_{h} \times \mathcal{V}_{h}
$$

As in the continuous case, this problem decomposes into a sequence of three wellposed problems, which are the respective discretizations of (3.2)-(3.4):

$$
\begin{aligned}
\phi_{h} \in \mathcal{V}_{h}: & \int_{\Omega} \nabla \phi_{h} \cdot \nabla v_{h}=-\int_{\Omega}(\boldsymbol{\eta} \nabla f) \cdot \nabla v_{h} \quad \forall v_{h} \in \mathcal{V}_{h}, \\
\boldsymbol{\psi}_{h} \in \mathcal{H}_{h}: & A\left(\boldsymbol{\psi}_{h}, \boldsymbol{\xi}_{h}\right)=G^{\phi_{h}}\left(\boldsymbol{\xi}_{h}\right) \quad \forall \boldsymbol{\xi}_{h} \in \mathcal{H}_{h}, \\
u_{h} \in \mathcal{V}_{h}: & \int_{\Omega} \nabla u_{h} \cdot \nabla \gamma_{h}=R^{\phi_{h}, \boldsymbol{\psi}_{h}}\left(\gamma_{h}\right) \quad \forall \gamma_{h} \in \mathcal{V}_{h} .
\end{aligned}
$$


Also, as in the continuous case, $\lambda_{h}$ is an eigenvalue of problem (3.7) if and only if $\mu_{h}:=\frac{1}{\lambda_{h}}$ is a non-zero eigenvalue of $T_{h}$ with the same multiplicity and corresponding eigenfunctions $u_{h}$.

Remark 3.5. The same arguments leading to Remark 2.9] allow us to show that any solution of problem (3.7) satisfies $\lambda_{h} \neq 0$. Moreover, $\int_{\Omega}\left(\boldsymbol{\eta} \nabla u_{h}\right) \cdot \nabla u_{h} \neq 0$ also holds true, but the proof of this fact is postponed to the Appendix (cf. Remark6.3. below).

In what follows we will prove that $T_{h} \rightarrow T$ in norm as $h \rightarrow 0$. As a consequence, for all non-zero $\mu \in \operatorname{sp}(T)$ and $h$ small enough, there exists $\mu_{h} \in \operatorname{sp}\left(T_{h}\right)$ such that $\mu_{h} \rightarrow \mu$. In particular, this implies that the discrete spectral problem (3.7) has solutions, at least for $h$ sufficiently small, as long as $\operatorname{sp}(T) \neq\{0\}$. A thorough spectral characterization is postponed to the Appendix (cf. Proposition 6.2. below), where the matrix form of problem (3.7) is introduced.

The following lemma yields the uniform convergence of $T_{h}$ to $T$ as $h \rightarrow 0$.

Lemma 3.6. There exist $C>0$ and $r \in\left(\frac{1}{2}, 1\right]$ such that, for all $f \in \mathcal{V}$,

$$
\left\|\left(T-T_{h}\right) f\right\|_{1, \Omega} \leq C h^{r}\|f\|_{1, \Omega} .
$$

Proof. Given $f \in \mathcal{V}$, let $(\phi, \boldsymbol{\psi}, u)$ and $\left(\phi_{h}, \boldsymbol{\psi}_{h}, u_{h}\right)$ be the solutions of problems (3.1) and (3.8), respectively, so that $u=T f$ and $u_{h}=T_{h} f$. From (3.4), (3.11), and the first Strang Lemma (cf. [7]), we have

$$
\left\|u-u_{h}\right\|_{1, \Omega} \leq C\left[\inf _{\gamma_{h} \in \mathcal{V}_{h}}\left\|u-\gamma_{h}\right\|_{1, \Omega}+\sup _{\gamma_{h} \in \mathcal{V}_{h}} \frac{R^{\phi_{h}, \boldsymbol{\psi}_{h}}\left(\gamma_{h}\right)-R^{\phi, \boldsymbol{\psi}}\left(\gamma_{h}\right)}{\left\|\gamma_{h}\right\|_{1, \Omega}}\right]
$$

To estimate the first term on the right-hand side above, we use standard approximation results and the regularity of $u$ proved in Lemma 3.2 .

$$
\inf _{\gamma_{h} \in \mathcal{V}_{h}}\left\|u-\gamma_{h}\right\|_{1, \Omega} \leq C h\|u\|_{2, \Omega} \leq C h\|f\|_{1, \Omega} .
$$

For the second term, we use the definition of $R$ (cf. (3.4)) and integration by parts to obtain

$$
\sup _{\gamma_{h} \in \mathcal{V}_{h}} \frac{R^{\phi_{h}, \boldsymbol{\psi}_{h}}\left(\gamma_{h}\right)-R^{\phi, \boldsymbol{\psi}}\left(\gamma_{h}\right)}{\left\|\gamma_{h}\right\|_{1, \Omega}} \leq C\left(\left\|\boldsymbol{\psi}-\boldsymbol{\psi}_{h}\right\|_{0, \Omega}+\left\|\phi-\phi_{h}\right\|_{0, \Omega}\right) .
$$

Now, we resort to a duality argument to estimate $\left\|\phi-\phi_{h}\right\|_{0, \Omega}$, since no additional regularity holds for $\phi$ (cf. Remark 3.4). Let

$$
\chi \in H^{1}(\Omega): \quad \int_{\Omega} \nabla \chi \cdot \nabla \gamma=\int_{\Omega}\left(\phi-\phi_{h}\right) \gamma \quad \forall \gamma \in H_{0}^{1}(\Omega) .
$$

By virtue of standard regularity results for the Laplace equation (see [12]), there exists $r \in\left(\frac{1}{2}, 1\right]$ such that $\chi \in H^{1+r}(\Omega)$ and

$$
\|\chi\|_{1+r, \Omega} \leq C\left\|\phi-\phi_{h}\right\|_{0, \Omega} \text {. }
$$

Let $\chi^{\mathrm{I}} \in \mathcal{V}_{h}$ be the Lagrange interpolant of $\chi$. Taking $\gamma=\phi-\phi_{h}$ in (3.15) and using (3.2), (3.9), and standard approximation results, we have

$$
\begin{aligned}
\left\|\phi-\phi_{h}\right\|_{0, \Omega}^{2} & =\int_{\Omega} \nabla \chi \cdot \nabla\left(\phi-\phi_{h}\right)=\int_{\Omega} \nabla\left(\chi-\chi^{\mathrm{I}}\right) \cdot \nabla\left(\phi-\phi_{h}\right) \\
& \leq C h^{r}\|\chi\|_{1+r, \Omega}\left\|\nabla\left(\phi-\phi_{h}\right)\right\|_{0, \Omega} \\
& \leq C h^{r}\left\|\phi-\phi_{h}\right\|_{0, \Omega}\left\|\nabla\left(\phi-\phi_{h}\right)\right\|_{0, \Omega} .
\end{aligned}
$$


Therefore, from (3.2) and (3.9), again, and (2.5),

$$
\left\|\phi-\phi_{h}\right\|_{0, \Omega} \leq C h^{r}\left(\|\nabla \phi\|_{0, \Omega}+\left\|\nabla \phi_{h}\right\|_{0, \Omega}\right) \leq C h^{r}\|f\|_{1, \Omega} .
$$

The next step is to estimate $\left\|\boldsymbol{\psi}-\boldsymbol{\psi}_{h}\right\|_{0, \Omega}$. With this aim, we first consider $\left\|\boldsymbol{\psi}-\boldsymbol{\psi}_{h}\right\|_{1, \Omega}$. From (3.3), (3.10), the ellipticity of $A$, and the first Strang Lemma again, we have

$$
\left\|\boldsymbol{\psi}-\boldsymbol{\psi}_{h}\right\|_{1, \Omega} \leq C\left[\inf _{\boldsymbol{\xi}_{h} \in \mathcal{H}_{h}}\left\|\boldsymbol{\psi}-\boldsymbol{\xi}_{h}\right\|_{1, \Omega}+\sup _{\boldsymbol{\xi}_{h} \in \mathcal{H}_{h}} \frac{G^{\phi_{h}}\left(\boldsymbol{\xi}_{h}\right)-G^{\phi}\left(\boldsymbol{\xi}_{h}\right)}{\left\|\boldsymbol{\xi}_{h}\right\|_{1, \Omega}}\right] .
$$

We use standard approximation results and Lemma 3.2 once more to obtain

$$
\inf _{\boldsymbol{\xi}_{h} \in \mathcal{H}_{h}}\left\|\boldsymbol{\psi}-\boldsymbol{\xi}_{h}\right\|_{1, \Omega} \leq C h^{s}\|\boldsymbol{\psi}\|_{1+s, \Omega} \leq C h^{s}\|f\|_{1, \Omega},
$$

with $s \in\left(\frac{1}{2}, 1\right]$, whereas from the definition of $G$ (cf. (3.3) $)$,

$$
\sup _{\boldsymbol{\xi}_{h} \in \mathcal{H}_{h}} \frac{G^{\phi_{h}}\left(\boldsymbol{\xi}_{h}\right)-G^{\phi}\left(\boldsymbol{\xi}_{h}\right)}{\left\|\boldsymbol{\xi}_{h}\right\|_{1, \Omega}} \leq C\left\|\phi-\phi_{h}\right\|_{0, \Omega} \leq C h^{r}\|f\|_{1, \Omega} .
$$

Thus, defining $t:=\min \{s, r\} \in\left(\frac{1}{2}, 1\right]$, we obtain that

$$
\left\|\boldsymbol{\psi}-\boldsymbol{\psi}_{h}\right\|_{1, \Omega} \leq C h^{t}\|f\|_{1, \Omega} .
$$

Next, we use another duality argument to estimate $\left\|\boldsymbol{\psi}-\boldsymbol{\psi}_{h}\right\|_{0, \Omega}$. Let

$$
\boldsymbol{\rho} \in \mathcal{H}: \quad A(\boldsymbol{\rho}, \boldsymbol{\xi})=\int_{\Omega}\left(\boldsymbol{\psi}-\boldsymbol{\psi}_{h}\right) \cdot \boldsymbol{\xi} \quad \forall \boldsymbol{\xi} \in \mathcal{H} .
$$

The same arguments used in the proof of Lemma 3.2 allow us to show that

$$
\|\boldsymbol{\rho}\|_{1+s, \Omega} \leq C\left\|\boldsymbol{\psi}-\boldsymbol{\psi}_{h}\right\|_{0, \Omega} .
$$

Hence, again using standard approximation results, we know that there exists $\boldsymbol{\rho}_{h} \in$ $\mathcal{H}_{h}$ such that

$$
\left\|\boldsymbol{\rho}-\boldsymbol{\rho}_{h}\right\|_{1, \Omega} \leq C h^{s}\|\boldsymbol{\rho}\|_{1+s, \Omega} \leq C h^{s}\left\|\boldsymbol{\psi}-\boldsymbol{\psi}_{h}\right\|_{0, \Omega} .
$$

Thus, taking $\boldsymbol{\xi}=\boldsymbol{\psi}-\boldsymbol{\psi}_{h}$ in (3.17) and using (3.3) and (3.10), we obtain

$$
\begin{aligned}
\left\|\boldsymbol{\psi}-\boldsymbol{\psi}_{h}\right\|_{0, \Omega}^{2} & =A\left(\boldsymbol{\rho}, \boldsymbol{\psi}-\boldsymbol{\psi}_{h}\right)=A\left(\boldsymbol{\rho}-\boldsymbol{\rho}_{h}, \boldsymbol{\psi}-\boldsymbol{\psi}_{h}\right)+A\left(\boldsymbol{\rho}_{h}, \boldsymbol{\psi}-\boldsymbol{\psi}_{h}\right) \\
& \leq C\left\|\boldsymbol{\rho}-\boldsymbol{\rho}_{h}\right\|_{1, \Omega}\left\|\boldsymbol{\psi}-\boldsymbol{\psi}_{h}\right\|_{1, \Omega}+\frac{1}{1+\nu}\left|\int_{\Omega}\left(\phi-\phi_{h}\right) \operatorname{curl} \boldsymbol{\rho}_{h}\right| \\
& \leq C h^{s+t}\left\|\boldsymbol{\psi}-\boldsymbol{\psi}_{h}\right\|_{0, \Omega}\|f\|_{1, \Omega}+C h^{r}\|f\|_{1, \Omega}\left\|\boldsymbol{\psi}-\boldsymbol{\psi}_{h}\right\|_{0, \Omega} .
\end{aligned}
$$

Therefore, since $s+t>1$,

$$
\left\|\boldsymbol{\psi}-\boldsymbol{\psi}_{h}\right\|_{0, \Omega} \leq C h^{r}\|f\|_{1, \Omega} .
$$

Thus, the lemma follows from (3.12), (3.13), (3.14), (3.16), and (3.18).

Remark 3.7. The order of convergence $r$ depends on the maximum Sobolev regularity of the domain for the Laplace equations with the right-hand side in $L^{2}(\Omega)$ and with homogeneous Dirichlet boundary conditions. In particular, if $\Omega$ is convex, then $r=1$. Otherwise, the lemma holds for all $r<r_{0}:=\frac{\pi}{\theta}$, with $\theta$ being the largest re-entrant angle of $\Omega$ (cf. [12]).

The following lemma shows that the error estimate for $\left\|\left(T-T_{h}\right) f\right\|_{1, \Omega}$ can be improved when $f$ is smoother. 
Lemma 3.8. There exists $C>0$ such that, for all $f \in \mathcal{V} \cap H^{2}(\Omega)$,

$$
\left\|\left(T-T_{h}\right) f\right\|_{1, \Omega} \leq C h\|f\|_{2, \Omega} .
$$

Proof. We follow exactly the same steps as in the proof of Lemma 3.6. However, now $\phi \in H^{1+r}(\Omega)$, with $r>\frac{1}{2}$ as in Remark 3.7 and $\|\phi\|_{1+r} \leq C\|f\|_{2, \Omega}$. In fact, $\phi$ is the solution of (3.2), which by virtue of Lemma 2.1 is a weak form of

$$
\begin{cases}-\Delta \phi=\boldsymbol{\eta}: D^{2} f \in L^{2}(\Omega) & \text { on } \Gamma .\end{cases}
$$

Hence, the estimate for $\left\|\phi-\phi_{h}\right\|_{0, \Omega}$ can be improved by using the fact that

$$
\left\|\nabla\left(\phi-\phi_{h}\right)\right\|_{0, \Omega} \leq C h^{r}\|\phi\|_{1+r, \Omega} \leq C h^{r}\|f\|_{2, \Omega} .
$$

Consequently, instead of (3.16) we obtain

$$
\left\|\phi-\phi_{h}\right\|_{0, \Omega} \leq C h^{2 r}\|f\|_{2, \Omega} .
$$

This last inequality can be used to improve (3.18) as follows:

$$
\left\|\boldsymbol{\psi}-\boldsymbol{\psi}_{h}\right\|_{0, \Omega} \leq C h^{s+t}\|f\|_{1, \Omega}+C h^{2 r}\|f\|_{2, \Omega} .
$$

Therefore, since $s+t>1$ and $2 r>1$, too, the lemma follows from (3.12), (3.13), (3.14), (3.19), and (3.20).

3.2. Spectral convergence and error estimates. As a direct consequence of Lemma 3.6. $T_{h}$ converges in norm to $T$ as $h$ goes to zero. Hence, standard results of spectral approximation (see, for instance, [15]) show that isolated parts of $\operatorname{sp}(T)$ are approximated by isolated parts of $\operatorname{sp}\left(T_{h}\right)$. More precisely, let $\mu \neq 0$ be an eigenvalue of $T$ with multiplicity $m$ and let $\mathcal{E}$ be its associated eigenspace. There exist $m$ eigenvalues $\mu_{h}^{(1)}, \ldots, \mu_{h}^{(m)}$ of $T_{h}$ (repeated according to their respective multiplicities) which converge to $\mu$. Let $\mathcal{E}_{h}$ be the direct sum of their corresponding associated eigenspaces.

We recall the definition of the gap $\hat{\delta}$ between two closed subspaces $\mathcal{M}$ and $\mathcal{N}$ of $H_{0}^{1}(\Omega)$ :

$$
\hat{\delta}(\mathcal{M}, \mathcal{N}):=\max \{\delta(\mathcal{M}, \mathcal{N}), \delta(\mathcal{N}, \mathcal{M})\}
$$

where

$$
\delta(\mathcal{M}, \mathcal{N}):=\sup _{\substack{x \in \mathcal{M} \\\|x\|_{1, \Omega}=1}}\left(\inf _{y \in \mathcal{N}}\|x-y\|_{1, \Omega}\right) .
$$

The following theorem implies spectral convergence with an optimal order for the approximation of the eigenfunctions.

Theorem 3.9. There exists a strictly positive constant $C$ such that

$$
\begin{aligned}
\hat{\delta}\left(\mathcal{E}, \mathcal{E}_{h}\right) & \leq C h, \\
\left|\mu-\mu_{h}^{(i)}\right| & \leq C h, \quad i=1, \ldots, m .
\end{aligned}
$$

Proof. As a consequence of Lemma 3.6 $T_{h}$ converges in norm to $T$ as $h$ goes to zero. Then, the proof follows as a direct consequence of Lemma 3.8 and Theorems 7.1 and 7.3 from 3 and the fact that, for $f \in \mathcal{E},\|f\|_{2, \Omega} \leq C\|f\|_{1, \Omega}$, because of Lemma 3.2 
The error estimates for the eigenvalues $\mu \neq 0$ of $T$ yield analogous estimates for the eigenvalues $\lambda=\frac{1}{\mu}$ of problem (2.21). However, the order of convergence in Theorem 3.9 is not optimal for $\mu$. Our next goal is to improve this order.

With this purpose, let us denote $\lambda_{h}:=1 / \mu_{h}^{(i)}$, with $\mu_{h}^{(i)}$ being any particular eigenvalue of $T_{h}$ converging to $\mu$. Let $u_{h}, \phi_{h}$, and $\boldsymbol{\psi}_{h}$ be such that $\left(\lambda_{h}, \phi_{h}, \boldsymbol{\psi}_{h}, u_{h}\right)$ is a solution of problem (3.7) with $\left\|u_{h}\right\|_{1, \Omega}=1$. According to Theorem 3.9, there exists a solution $(\lambda, \phi, \boldsymbol{\psi}, u)$ of problem (2.21) with $\|u\|_{1, \Omega}=1$ such that

$$
\left\|u-u_{h}\right\|_{1, \Omega} \leq C h \text {. }
$$

The following lemma, which will be used to prove an improved order of convergence for the corresponding eigenvalues, shows estimates for $\phi-\phi_{h}$ and $\boldsymbol{\psi}-\boldsymbol{\psi}_{h}$.

Lemma 3.10. There exists $C>0$ such that

$$
\begin{aligned}
\left\|\phi-\phi_{h}\right\|_{1, \Omega}+\left\|\boldsymbol{\psi}-\boldsymbol{\psi}_{h}\right\|_{1, \Omega} & \leq C\left(h+\inf _{v_{h} \in \mathcal{V}_{h}}\left\|\phi-v_{h}\right\|_{1, \Omega}+\inf _{\boldsymbol{\xi}_{h} \in \mathcal{H}_{h}}\left\|\boldsymbol{\psi}-\boldsymbol{\xi}_{h}\right\|_{1, \Omega}\right) \\
& \leq C h^{t},
\end{aligned}
$$

where $t:=\min \{s, r\} \in\left(\frac{1}{2}, 1\right]$, with $s$ and $r$ as in Lemmas 3.2 and 3.6 , respectively.

Proof. First note that $(\phi, \boldsymbol{\psi}, u)$ is the solution of problem 3.1 with $f=\lambda u$. Hence, from Lemma 3.2 $u \in H^{2}(\Omega)$ with $\|u\|_{2, \Omega} \leq C \lambda\|u\|_{1, \Omega}$. Hence, the same arguments used in the proof of Lemma 3.8 allow us to show that

$$
\|\phi\|_{1+r, \Omega} \leq C\|u\|_{2, \Omega} \leq C \lambda\|u\|_{1, \Omega} .
$$

On the other hand, $\left(\phi_{h}, \boldsymbol{\psi}_{h}, u_{h}\right)$ is the solution of problem (3.8) with $f=\lambda_{h} u_{h}$. Thus, from the equivalence between this problem and (3.9)-(3.11), $\phi_{h}$ is the solution of (3.9) with $f=\lambda_{h} u_{h}$. Hence, from the first Strang Lemma again,

$$
\left\|\phi-\phi_{h}\right\|_{1, \Omega} \leq C\left[\inf _{v_{h} \in \mathcal{V}_{h}}\left\|\phi-v_{h}\right\|_{1, \Omega}+\sup _{v_{h} \in \mathcal{V}_{h}} \frac{\int_{\Omega}\left[\boldsymbol{\eta}\left(\lambda \nabla u-\lambda_{h} \nabla u_{h}\right)\right] \cdot \nabla v_{h}}{\left\|v_{h}\right\|_{1, \Omega}}\right] .
$$

To estimate the first term on the right-hand side above, we use standard approximation results:

$$
\inf _{v_{h} \in \mathcal{V}_{h}}\left\|\phi-v_{h}\right\|_{1, \Omega} \leq C h^{r}\|\phi\|_{1+r, \Omega} \leq C h^{r}\|u\|_{1, \Omega} .
$$

For the second term, we use the Cauchy-Schwarz inequality, (2.5), (3.21), and Theorem 3.9 .

$$
\begin{aligned}
\sup _{v_{h} \in \mathcal{V}_{h}} \frac{\int_{\Omega}\left[\boldsymbol{\eta}\left(\lambda \nabla u-\lambda_{h} \nabla u_{h}\right)\right] \cdot \nabla v_{h}}{\left\|v_{h}\right\|_{1, \Omega}} & \leq C\left\|\lambda \nabla u-\lambda_{h} \nabla u_{h}\right\|_{0, \Omega} \\
& \leq C|\lambda|\left\|u-u_{h}\right\|_{1, \Omega}+\left|\lambda-\lambda_{h}\right|\left\|u_{h}\right\|_{1, \Omega} \\
& \leq C h .
\end{aligned}
$$

On the other hand, to estimate the term $\left\|\boldsymbol{\psi}-\boldsymbol{\psi}_{h}\right\|_{1, \Omega}$, we repeat the arguments in the proof of Lemma 3.6 (with $f=\lambda u$ ) to obtain

$$
\begin{aligned}
\left\|\boldsymbol{\psi}-\boldsymbol{\psi}_{h}\right\|_{1, \Omega} & \leq C\left(\inf _{\boldsymbol{\xi}_{h} \in \mathcal{H}_{h}}\left\|\boldsymbol{\psi}-\boldsymbol{\xi}_{h}\right\|_{1, \Omega}+\left\|\phi-\phi_{h}\right\|_{0, \Omega}\right) \\
& \leq C h^{s} \lambda\|u\|_{1, \Omega}+C\left\|\phi-\phi_{h}\right\|_{0, \Omega} .
\end{aligned}
$$


Next, repeating the arguments in the proof of Lemma 3.8 we have from (3.19) that

$$
\left\|\phi-\phi_{h}\right\|_{0, \Omega} \leq C h^{2 r} \lambda\|u\|_{1, \Omega} .
$$

Thus, we conclude the proof.

Now we are in a position to prove an improved order of convergence for the eigenvalues.

Theorem 3.11. There exists a strictly positive constant $C$ such that

$$
\left|\lambda-\lambda_{h}\right| \leq C\left(h^{2}+\inf _{v_{h} \in \mathcal{V}_{h}}\left\|\phi-v_{h}\right\|_{1, \Omega}^{2}+\inf _{\boldsymbol{\xi}_{h} \in \mathcal{H}_{h}}\left\|\boldsymbol{\psi}-\boldsymbol{\xi}_{h}\right\|_{1, \Omega}^{2}\right) \leq C h^{2 t},
$$

with $t \in\left(\frac{1}{2}, 1\right]$ as in Lemma 3.10 .

Proof. We adapt to our case a standard argument (cf. 33, Lemma 9.1]). Let $U:=$ $(\phi, \boldsymbol{\psi}, u)$ and $U_{h}:=\left(\phi_{h}, \boldsymbol{\psi}_{h}, u_{h}\right)$ be as in the proof of Lemma 3.10 Because of (2.21) and (3.7),

$$
\begin{aligned}
\mathscr{A}\left(U-U_{h}, U-U_{h}\right) & =\mathscr{A}(U, U)-2 \mathscr{A}\left(U, U_{h}\right)+\mathscr{A}\left(U_{h}, U_{h}\right) \\
& =-\lambda \mathscr{C}(u, u)+2 \lambda \mathscr{C}\left(u, u_{h}\right)-\lambda_{h} \mathscr{C}\left(u_{h}, u_{h}\right),
\end{aligned}
$$

whereas

$$
\lambda \mathscr{C}\left(u-u_{h}, u-u_{h}\right)=\lambda \mathscr{C}(u, u)-2 \lambda \mathscr{C}\left(u, u_{h}\right)+\lambda \mathscr{C}\left(u_{h}, u_{h}\right) .
$$

Therefore, since $\mathscr{C}\left(u_{h}, u_{h}\right) \neq 0$ (cf. Remark 3.5),

$$
\lambda-\lambda_{h}=\frac{\mathscr{A}\left(U-U_{h}, U-U_{h}\right)+\lambda \mathscr{C}\left(u-u_{h}, u-u_{h}\right)}{\mathscr{C}\left(u_{h}, u_{h}\right)} .
$$

Moreover, from (3.21), $\mathscr{C}\left(u_{h}, u_{h}\right) \stackrel{h}{\rightarrow} \mathscr{C}(u, u) \neq 0$ (cf. Remark 2.9) . Hence,

$$
\begin{aligned}
\left|\lambda-\lambda_{h}\right| & \leq C\left(\left|\mathscr{A}\left(U-U_{h}, U-U_{h}\right)\right|+|\lambda|\left|\mathscr{C}\left(u-u_{h}, u-u_{h}\right)\right|\right) \\
& \leq C\left(\left\|U-U_{h}\right\|_{\mathcal{V} \times \mathcal{H} \times \mathcal{V}}^{2}+\left\|u-u_{h}\right\|_{1, \Omega}^{2}\right) \\
& \leq C\left(h^{2}+\inf _{v_{h} \in \mathcal{V}_{h}}\left\|\phi-v_{h}\right\|_{1, \Omega}^{2}+\inf _{\boldsymbol{\xi}_{h} \in \mathcal{H}_{h}}\left\|\boldsymbol{\psi}-\boldsymbol{\xi}_{h}\right\|_{1, \Omega}^{2}\right) \\
& \leq C h^{2 t},
\end{aligned}
$$

the last two inequalities because of (3.21) and Lemma 3.10. Thus, we conclude the proof.

Remark 3.12. The order of convergence for the eigenvalues does not depend on the regularity of the eigenfunction $u$, which always belongs to $H^{2}(\Omega)$, but on the regularity of the auxiliary quantities $\phi$ and $\boldsymbol{\psi}$. In fact, the $\mathcal{O}\left(h^{t}\right)$ error estimate in Lemma 3.10 could be improved, provided $\phi$ and $\boldsymbol{\psi}$ were more regular.

\section{Numerical analysis of the Vibration PROBlem}

In this section we summarize the results for the vibration problem. We do not include most of the proofs since they are either similar to the corresponding ones for the buckling problem or simpler. We only emphasize those aspects that differ from the buckling problem.

Consider the well-posed source problem associated with the vibration problem (2.22): 
Given $f \in L^{2}(\Omega)$, find $(\phi, \boldsymbol{\psi}, u) \in \mathcal{V} \times \mathcal{H} \times \mathcal{V}$ such that

$$
\mathscr{A}((\phi, \boldsymbol{\psi}, u),(\gamma, \boldsymbol{\xi}, v))=-\mathscr{B}(f, v) \quad \forall(\gamma, \boldsymbol{\xi}, v) \in \mathcal{V} \times \mathcal{H} \times \mathcal{V}
$$

Let $T$ be the bounded linear operator defined by

$$
\begin{aligned}
T: L^{2}(\Omega) & \rightarrow L^{2}(\Omega), \\
f & \mapsto u,
\end{aligned}
$$

with $(\phi, \boldsymbol{\psi}, u) \in \mathcal{V} \times \boldsymbol{H} \times \mathcal{V}$ being the solution of (4.1). Clearly $\lambda$ is an eigenvalue of problem (2.22) if and only if $\mu:=\frac{1}{\lambda}$ is a non-zero eigenvalue of $T$, with the same multiplicity and corresponding eigenfunctions $u$ (recall $\lambda \neq 0$; cf. Remark 2.9).

For the vibration problem, the operator $T$ is self-adjoint with respect to the $L^{2}(\Omega)$ inner product. Moreover $T$ is compact, because of the compact inclusion $\mathcal{V} \hookrightarrow L^{2}(\Omega)$, and the following spectral characterization holds:

Lemma 4.1. The spectrum of $T$ satisfies $\operatorname{sp}(T)=\{0\} \cup\left\{\mu_{n}: n \in \mathbb{N}\right\}$, where $\left\{\mu_{n}\right\}_{n \in \mathbb{N}}$ is a sequence of real positive eigenvalues which converges to 0 . The multiplicity of each eigenvalue is finite, and its ascent is 1.

The following additional regularity result holds true in this case:

Lemma 4.2. There exist $r, s \in\left(\frac{1}{2}, 1\right]$ and $C>0$ such that, for all $f \in L^{2}(\Omega)$, the solution $(\phi, \boldsymbol{\psi}, u)$ of problem (4.1) satisfies $\phi \in H^{1+r}(\Omega), u \in H^{2+s}(\Omega), \psi \in$ $H^{1+s}(\Omega)^{2}$, and

$$
\|\phi\|_{1+r, \Omega}+\|u\|_{2+s, \Omega}+\|\boldsymbol{\psi}\|_{1+s, \Omega} \leq C\|f\|_{0, \Omega} .
$$

Constants $r$ and $s$ above are the same as those in the proof of Lemmas 3.2 and 3.6. By comparing this result with Lemma 3.2, we observe that $\phi$ is smoother in this case than for the buckling problem. This is the key point which makes the analysis of the vibration problem a bit simpler.

4.1. Finite element approximation. The discrete version of problem (2.22) reads as follows:

Find $\left(\lambda_{h}, \phi_{h}, \psi_{h}, u_{h}\right) \in \mathbb{R} \times \mathcal{V}_{h} \times \mathcal{H}_{h} \times \mathcal{V}_{h}, u_{h} \neq 0$, such that

$$
\mathscr{A}\left(\left(\phi_{h}, \boldsymbol{\psi}_{h}, u_{h}\right),\left(\gamma_{h}, \boldsymbol{\xi}_{h}, v_{h}\right)\right)=-\lambda_{h} \mathscr{B}\left(u_{h}, v_{h}\right) \quad \forall\left(\gamma_{h}, \boldsymbol{\xi}_{h}, v_{h}\right) \in \mathcal{V}_{h} \times \mathcal{H}_{h} \times \mathcal{V}_{h}
$$

Let $T_{h}$ be the bounded linear operator defined by

$$
\begin{aligned}
T_{h}: L^{2}(\Omega) & \rightarrow L^{2}(\Omega), \\
f & \mapsto u_{h},
\end{aligned}
$$

with $\left(\phi_{h}, \boldsymbol{\psi}_{h}, u_{h}\right) \in \mathcal{V}_{h} \times \mathcal{H}_{h} \times \mathcal{V}_{h}$ being the solution of

Once more, $\lambda_{h}$ is an eigenvalue of problem (4.2) if and only if $\mu_{h}:=\frac{1}{\lambda_{h}}$ is a nonzero eigenvalue of $T_{h}$, with the same multiplicity and corresponding eigenfunctions $u_{h}$. Also, as in the continuous case, $\lambda_{h} \neq 0$.

In this case, $T_{h}$ is self-adjoint with respect to the $L^{2}(\Omega)$ inner product. Because of this, it is easy to prove the following spectral characterization:

Lemma 4.3. Problem (4.2) has exactly $\operatorname{dim} \mathcal{V}_{h}$ eigenvalues, repeated accordingly to their respective multiplicities. All of them are real and positive. 
The following lemma yields the uniform convergence of $T_{h}$ to $T$ as $h \rightarrow 0$. Its proof follows the lines of the proof of Lemma 3.8 by taking advantage of the additional regularity of $\phi$ (cf. Lemma 4.2).

Lemma 4.4. There exists $C>0$ such that, for all $f \in L^{2}(\Omega)$,

$$
\left\|\left(T-T_{h}\right) f\right\|_{1, \Omega} \leq C h\|f\|_{0, \Omega} \text {. }
$$

4.2. Spectral convergence and error estimates. As a direct consequence of Lemma 4.4, $T_{h}$ converges in $H^{1}(\Omega)$ norm to $T$ as $h$ goes to zero (as well as in $L^{2}(\Omega$ ) norm). Hence, isolated parts of $\operatorname{sp}(T)$ are approximated by isolated parts of $\operatorname{sp}\left(T_{h}\right)$. Let $\mu \neq 0$ be an eigenvalue of $T$ with multiplicity $m$ and let $\mathcal{E}$ be its associated eigenspace. There exist $m$ eigenvalues $\mu_{h}^{(1)}, \ldots, \mu_{h}^{(m)}$ of $T_{h}$ (repeated according to their respective multiplicities) which converge to $\mu$. Let $\mathcal{E}_{h}$ be the direct sum of their corresponding associated eigenspaces. The following error estimate is again a direct consequence of standard spectral approximation results (cf. [3] ):

Theorem 4.5. There exists a strictly positive constant $C$ such that

$$
\hat{\delta}\left(\mathcal{E}, \mathcal{E}_{h}\right) \leq C h .
$$

Finally an improved order of convergence also holds for the eigenvalues. To prove this, we do not need to resort to the analog of Lemma 3.10. We include in this case the simpler proof of the following theorem, where, for each $f \in \mathcal{E}$, we denote by $U^{f}:=\left(\phi^{f}, \boldsymbol{\psi}^{f}, u^{f}\right)$ the solution of problem (4.1). (Notice that $u^{f}=T f=\mu f$.)

Theorem 4.6. There exists a strictly positive constant $C$ such that

$$
\begin{aligned}
\left|\mu-\mu_{h}^{(i)}\right| & \leq C\left[h+\sup _{f \in \mathcal{E}}\left(\frac{\inf _{v_{h} \in \mathcal{V}_{h}}\left\|\phi^{f}-v_{h}\right\|_{1, \Omega}+\inf _{\boldsymbol{\xi}_{h} \in \mathcal{H}_{h}}\left\|\boldsymbol{\psi}^{f}-\boldsymbol{\xi}_{h}\right\|_{1, \Omega}}{\|f\|_{0, \Omega}}\right)\right]^{2} \\
& \leq C h^{2 t}, \quad i=1, \ldots, m,
\end{aligned}
$$

where $t=\min \{s, r\}$, with $r, s \in\left(\frac{1}{2}, 1\right]$ as in Lemma 4.2.

Proof. By applying Theorem 7.3 from [3] and taking into account that $T$ and $T_{h}$ are self-adjoint with respect to the $L^{2}(\Omega)$ inner product, we have

$$
\left|\mu-\mu_{h}^{(i)}\right| \leq C\left[\sup _{f, g \in \mathcal{E}} \frac{\int_{\Omega}\left(T f-T_{h} f\right) g}{\|f\|_{0, \Omega}\|g\|_{0, \Omega}}+\sup _{f \in \mathcal{E}} \frac{\left\|\left(T-T_{h}\right) f\right\|_{0, \Omega}^{2}}{\|f\|_{0, \Omega}^{2}}\right], \quad i=1, \ldots, m .
$$

The second term on the right-hand side above is directly bounded by means of Lemma 4.4. so there only remains to estimate the first one. With this aim, let $f, g \in \mathcal{E}$. Let $U^{f}$ and $U^{g}$ be defined as above. Let $U_{h}^{f}:=\left(\phi_{h}^{f}, \boldsymbol{\psi}_{h}^{f}, u_{h}^{f}\right)$ and $U_{h}^{g}:=\left(\phi_{h}^{g}, \boldsymbol{\psi}_{h}^{g}, u_{h}^{g}\right)$ be the solutions of problem (4.3) with data $f$ and $g$, respectively. There holds

$$
\begin{aligned}
\int_{\Omega}\left(T f-T_{h} f\right) g & =\mathscr{B}\left(u^{f}-u_{h}^{f}, g\right)=-\mathscr{A}\left(U^{f}-U_{h}^{f}, U^{g}\right)=-\mathscr{A}\left(U^{f}-U_{h}^{f}, U^{g}-U_{h}^{g}\right) \\
& \leq C\left\|U^{f}-U_{h}^{f}\right\|_{\mathcal{V} \times \mathcal{H} \times \mathcal{V}}\left\|U^{g}-U_{h}^{g}\right\|_{\mathcal{V} \times \mathcal{H} \times \mathcal{V}},
\end{aligned}
$$

because of the standard Galerkin orthogonality and the continuity of $\mathscr{A}$. Now,

$$
\begin{aligned}
\left\|U^{f}-U_{h}^{f}\right\|_{\mathcal{V} \times \mathcal{H} \times \mathcal{V}} & \leq\left\|\phi^{f}-\phi_{h}^{f}\right\|_{1, \Omega}+\left\|\boldsymbol{\psi}^{f}-\boldsymbol{\psi}_{h}^{f}\right\|_{\mathcal{H}}+\left\|u^{f}-u_{h}^{f}\right\|_{1, \Omega} \\
& \leq \inf _{v_{h} \in \mathcal{V}_{h}}\left\|\phi^{f}-v_{h}\right\|_{1, \Omega}+\inf _{\boldsymbol{\xi}_{h} \in \mathcal{H}_{h}}\left\|\boldsymbol{\psi}^{f}-\boldsymbol{\xi}_{h}\right\|_{1, \Omega}+C h\|f\|_{0, \Omega} \\
& \leq C h^{t}\|f\|_{0, \Omega},
\end{aligned}
$$


where we have used results from [1, Section 5] to estimate the terms $\left\|\phi^{f}-\phi_{h}^{f}\right\|_{1, \Omega}$ and $\left\|\boldsymbol{\psi}^{f}-\boldsymbol{\psi}_{h}^{f}\right\|_{\mathcal{H}}$ and from Lemma 4.4 for $\left\|u^{f}-u_{h}^{f}\right\|_{1, \Omega}$. Since the same holds for

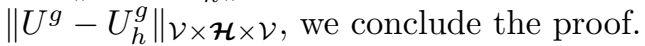

The error estimate from the previous lemma yields a similar one for the eigen-

values $\lambda=\frac{1}{\mu}$ of problem (2.22). Moreover, a remark analogous to Remark 3.12 also holds in this case.

\section{Numerical RESUlts}

We report in this section some numerical experiments which confirm the theoretical results proved above. Moreover, we compare in the first two tests the performance of the proposed method with those of the Ciarlet-Raviart [8, 6, 17] and Ishihara [13, 14] methods.

The Ciarlet-Raviart method is based on a mixed form of the biharmonic equation, which is equivalent to this equation for convex domains. This method was proved to converge for the vibration and the buckling problems for finite elements of degree $k \geq 2$ (see [17, Section $7(\mathrm{~b}, \mathrm{~d})]$ ). Our experiments will give evidence of optimal order convergence for piecewise linear finite elements, although, to the best of our knowledge, this has not been proved.

Ishihara's method is based on an alternative mixed formulation, also equivalent to the biharmonic equation for convex domains. Its piecewise linear discretization was analyzed in [13] for the vibration problem and in [14] for the buckling problem. It was proved that it converges in both cases, with a suboptimal order $\mathcal{O}\left(h^{1 / 2}\right)$, only for meshes which are uniform in the interior of the domain. Our numerical experiments will show that this constraint is not a technicality, since the method converges to wrong results when used on particular regular non-uniform meshes.

Since there is no significant difference in our experiments between the vibration and the buckling problems, we will only report the numerical results for the latter. We have taken in all our experiments a Poisson ratio $\nu=0.25$.

5.1. Test 1: Uniformly compressed square plate; uniform meshes. We have taken as an example of a convex domain the unit square $\Omega:=(0,1) \times(0,1)$. We have used the stress distribution corresponding to a uniformly compressed plate: $\boldsymbol{\eta}=\mathbf{I}$.

We have used uniform meshes as those shown in Figure 1, The refinement parameter $N$ used to label each mesh is the number of elements on each edge of the plate.

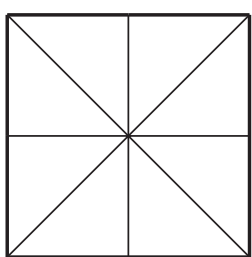

$N=2$

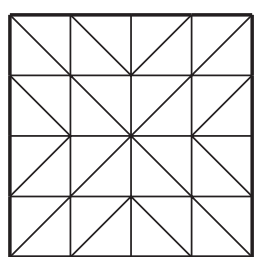

$N=4$

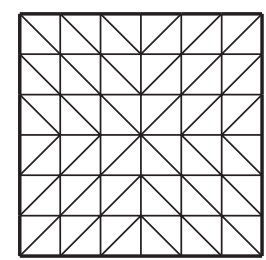

$N=6$

Figure 1. Square plate: uniform meshes. 
We report in Table 1 the lowest buckling coefficients (i.e., the lowest eigenvalues of the buckling problem) computed with the method analyzed in this paper, with Ciarlet-Raviart's method, and with Ishihara's method. The table includes computed orders of convergence and extrapolated more accurate values of each eigenvalue obtained by means of a least-squares fitting.

TABLE 1. Lowest buckling coefficients of a uniformly compressed clamped square plate computed on uniform meshes with the method analyzed in this paper (A), Ciarlet-Raviart's method (CR), and Ishihara's method (I).

\begin{tabular}{|c|c|c|c|c|c|c|c|}
\hline & Method & $N=24$ & $N=36$ & $N=48$ & $N=60$ & Order & Extrapolated \\
\hline \multirow{3}{*}{$\lambda_{1}$} & $\mathrm{~A}$ & 5.3051 & 5.3042 & 5.3039 & 5.3038 & 2.61 & 5.3037 \\
\hline & $\mathrm{CR}$ & 5.3830 & 5.3395 & 5.3239 & 5.3167 & 1.95 & 5.3033 \\
\hline & I & 5.3529 & 5.3254 & 5.3159 & 5.3114 & 2.02 & 5.3037 \\
\hline \multirow{3}{*}{$\lambda_{2}=\lambda_{3}$} & $\bar{A}$ & 9.3578 & 9.3444 & 9.3398 & 9.3378 & 2.09 & 9.3343 \\
\hline & CR & 9.5390 & 9.4261 & 9.3861 & 9.3675 & 1.97 & 9.3337 \\
\hline & I & 9.4650 & 9.3912 & 9.3659 & 9.3544 & 2.06 & 9.3347 \\
\hline \multirow{3}{*}{$\lambda_{4}$} & $\bar{A}$ & 13.0346 & 13.0091 & 13.0007 & 12.9969 & 2.14 & 12.9908 \\
\hline & CR & 13.3977 & 13.1710 & 13.0919 & 13.0553 & 2.01 & 12.9909 \\
\hline & I & 13.2128 & 13.0827 & 13.0407 & 13.0219 & 2.21 & 12.9930 \\
\hline
\end{tabular}

It can be seen from Table 1 that the three methods converge in this case to the same values with optimal quadratic order, although this has been proved only for the method analyzed in this paper (cf. Remark 3.7). Notice that, for all the methods, the second computed eigenvalue is double, because the meshes preserve the symmetry of the domain leading to an eigenvalue of multiplicity 2 in the continuous problem.

Figure 2 shows the transverse displacements of the principal buckling mode (i.e., the eigenfunction corresponding to the lowest eigenvalue of the buckling problem) computed with the method analyzed in this paper.

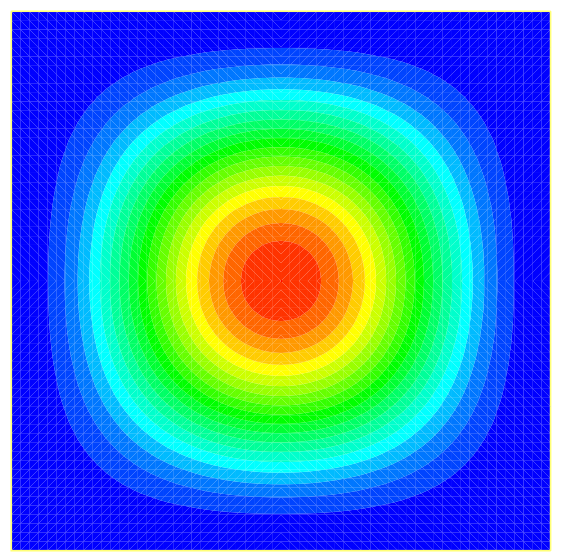

FigURE 2. Uniformly compressed square plate; principal buckling mode. 
5.2. Test 2: Uniformly compressed square plate; non-uniform meshes. We have tested the same three methods as above on non-uniform meshes, as well. We have solved the same problem as in the previous example with tiled meshes as those shown in Figure 3. The refinement parameter $N$ used to label each mesh is now the number of tiles on each edge of the plate. The reason for this choice is to avoid asymptotically uniform meshes.

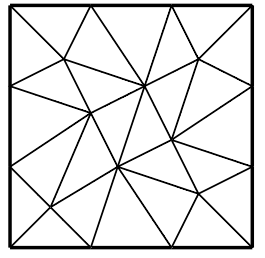

$N=1$

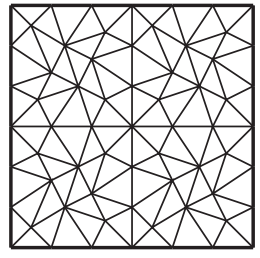

$N=2$

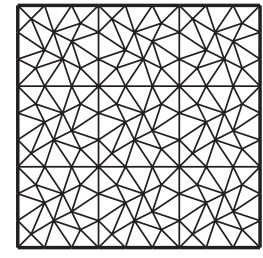

$N=3$

Figure 3. Square plate: tiled meshes.

We report in Table 2 the lowest buckling coefficients computed on these meshes with each of the three methods again. Notice that in this case, since the meshes do not preserve the symmetry of the domain, the second eigenvalue, which has multiplicity 2 in the continuous problem, will be in general approximated by two simple eigenvalues.

TABLE 2. Lowest buckling coefficients of a uniformly compressed clamped square plate computed on non-uniform meshes with the method analyzed in this paper (A), Ciarlet-Raviart's method (CR), and Ishihara's method (I).

\begin{tabular}{rcrrrrrc}
\hline & Method & $N=15$ & $N=25$ & $N=35$ & $N=45$ & Order & Extrapolated \\
\hline \multirow{3}{*}{$\lambda_{1}$} & $\mathrm{~A}$ & 5.3030 & 5.3034 & 5.3035 & 5.3036 & 1.91 & 5.3036 \\
& $\mathrm{CR}$ & 5.3134 & 5.3075 & 5.3058 & 5.3050 & 1.88 & 5.3038 \\
& $\mathrm{I}$ & 5.4491 & 5.4384 & 5.4348 & 5.4328 & 1.42 & 5.4285 \\
\hline \multirow{3}{*}{$\lambda_{2}$} & $\mathrm{~A}$ & 9.3323 & 9.3335 & 9.3338 & 9.3339 & 1.96 & 9.3342 \\
& $\mathrm{CR}$ & 9.3622 & 9.3449 & 9.3399 & 9.3379 & 1.92 & 9.3345 \\
& $\mathrm{I}$ & 9.5788 & 9.5520 & 9.5433 & 9.5388 & 1.56 & 9.5302 \\
\hline \multirow{4}{*}{$\lambda_{3}$} & $\mathrm{~A}$ & 9.3336 & 9.3340 & 9.3340 & 9.3341 & 1.79 & 9.3342 \\
& $\mathrm{CR}$ & 9.3641 & 9.3455 & 9.3402 & 9.3380 & 1.94 & 9.3345 \\
& $\mathrm{I}$ & 9.6337 & 9.6106 & 9.6030 & 9.5991 & 1.54 & 9.5914 \\
$\lambda_{4}$ & $\mathrm{~A}$ & 12.9830 & 12.9876 & 12.9890 & 12.9895 & 1.96 & 12.9904 \\
& $\mathrm{CR}$ & 13.0437 & 13.0103 & 13.0010 & 12.9970 & 1.95 & 12.9908 \\
& $\mathrm{I}$ & 13.3387 & 13.2949 & 13.2811 & 13.2743 & 1.64 & 13.2617 \\
\hline
\end{tabular}

It can be seen from Table 2 that the method analyzed in this paper and CiarletRaviart's method do not deteriorate on these meshes and converge to the same values with quadratic order again. Instead, this is not the case for Ishihara's method, which converges to wrong results. 
5.3. Test 3: Shear loaded square plate. For this test we have computed the buckling coefficients of the same plate as in the previous example, subjected to a uniform shear load. This corresponds to a plane stress field

$$
\boldsymbol{\eta}=\left(\begin{array}{ll}
0 & 1 \\
1 & 0
\end{array}\right)
$$

Note that $\boldsymbol{\eta}$ is not positive definite in this case.

We report in Table 3 the lowest buckling coefficients computed on the same uniform meshes used in Test 1 (cf. Figure 1) with the method analyzed in this paper.

TABLE 3. Lowest buckling coefficients of a shear loaded clamped square plate computed on uniform meshes with the method analyzed in this paper.

\begin{tabular}{ccccccc}
\hline & $N=24$ & $N=36$ & $N=48$ & $N=60$ & Order & Extrapolated \\
\hline$\lambda_{1}$ & 14.8218 & 14.7215 & 14.6867 & 14.6706 & 2.01 & 14.6420 \\
$\lambda_{2}$ & 17.3111 & 17.0922 & 17.0161 & 16.9810 & 2.02 & 16.9195 \\
$\lambda_{3}$ & 36.0905 & 34.5656 & 34.0304 & 33.7825 & 1.99 & 33.3376 \\
\hline
\end{tabular}

Once more, the method converges with optimal quadratic order. Although we do not report the results obtained with the other two methods, both converge on uniform meshes to the same eigenvalues.

Figure 4 shows the transverse displacements of the principal buckling mode for the shear loaded square plate computed with the method analyzed in this paper.

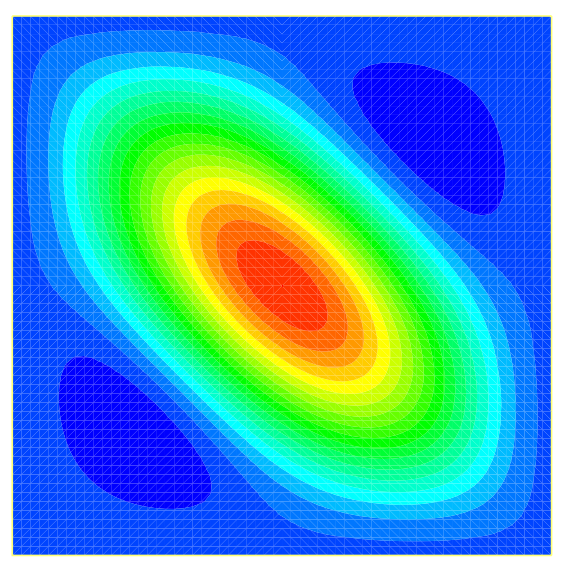

FiguRE 4. Shear loaded square plate; principal buckling mode.

5.4. Test 4: L-shaped plate. Finally, we have computed the buckling coefficients of an L-shaped plate: $\Omega:=(0,1) \times(0,1) \backslash[0.5,1) \times[0.5,1)$. We have used $\boldsymbol{\eta}=$ I (uniform compression) and uniform meshes as those shown in Figure 5 . The meaning of the refinement parameter $N$ is clear from this figure. 

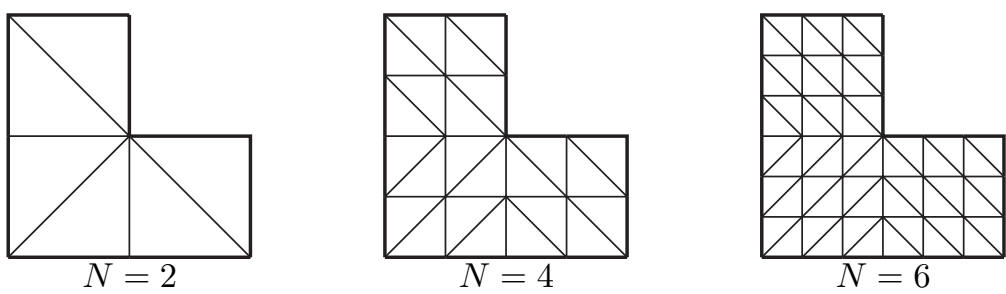

FiguRE 5. L-shaped plate: uniform meshes.

We report in Table 4 the lowest buckling coefficients computed with the method analyzed in this paper.

TABLE 4. Lowest buckling coefficients of an L-shaped clamped plate computed on uniform meshes with the method analyzed in this paper.

\begin{tabular}{ccccccc}
\hline & $N=40$ & $N=60$ & $N=80$ & $N=100$ & Order & Extrapolated \\
\hline$\lambda_{1}$ & 12.8379 & 12.9010 & 12.9328 & 12.9518 & 0.99 & 13.0290 \\
$\lambda_{2}$ & 14.9175 & 14.9586 & 14.9752 & 14.9838 & 1.60 & 15.0036 \\
$\lambda_{3}$ & 17.0083 & 16.9993 & 16.9968 & 16.9960 & 2.75 & 16.9949 \\
\hline
\end{tabular}

In this case, for the first buckling coefficient, the method converges with order close to 1.089 , which is the expected one because of the singularity of the solution (see [12]). Instead, the method converges with larger orders for the second and the third buckling coefficients.

Notice that, according to Theorem 3.11, the order of convergence for the buckling coefficients must double the worst among those of $\left\|\phi-\phi_{h}\right\|_{1, \Omega},\left\|\boldsymbol{\psi}-\boldsymbol{\psi}_{h}\right\|_{1, \Omega}$, and $\left\|u-u_{h}\right\|_{1, \Omega}$. In the case of $\lambda_{1}$ and $\lambda_{2}$, the worst order should be that of $\left\|\boldsymbol{\psi}-\boldsymbol{\psi}_{h}\right\|_{1, \Omega}$. In fact, according to (3.21), the transverse displacement $u$ satisfies $\left\|u-u_{h}\right\|_{1, \Omega}=$ $\mathcal{O}(h)$ for any polygonal domain $\Omega$. Moreover, since in this case $\boldsymbol{\eta}=\mathbf{I}$, we have $\phi=\lambda u$ and $\phi_{h}=\lambda_{h} u_{h}$, so that $\left\|\phi-\phi_{h}\right\|_{1, \Omega}=\mathcal{O}(h)$, too.

We include in Table 5 computed orders of convergence $\left\|u_{h}-u_{\mathrm{ex}}\right\|_{1, \Omega}$, where we have used as 'exact' transverse displacements $u_{\mathrm{ex}}$ the ones computed with a highly refined mesh corresponding to $N=200$.

TABLE 5. Errors of the transverse displacements $\left\|u_{h}-u_{\text {ex }}\right\|_{1, \Omega}$ for the lowest buckling coefficients of an L-shaped clamped plate computed on uniform meshes with the method analyzed in this paper.

\begin{tabular}{cccccc}
\hline & $N=8$ & $N=16$ & $N=24$ & $N=32$ & Order \\
\hline$\lambda_{1}$ & 0.4514 & 0.2297 & 0.1477 & 0.1059 & 1.04 \\
$\lambda_{2}$ & 0.4424 & 0.2218 & 0.1411 & 0.1005 & 1.07 \\
$\lambda_{3}$ & 0.5028 & 0.2469 & 0.1570 & 0.1121 & 1.08 \\
\hline
\end{tabular}

It can be seen from this table that the eigenfunctions $u_{h}$ actually converge with order $\mathcal{O}(h)$ as Theorem 3.9 predicts, in spite of the non-convex angle of the domain. 
Finally, Figure 6 shows the transverse displacement of the principal buckling mode.

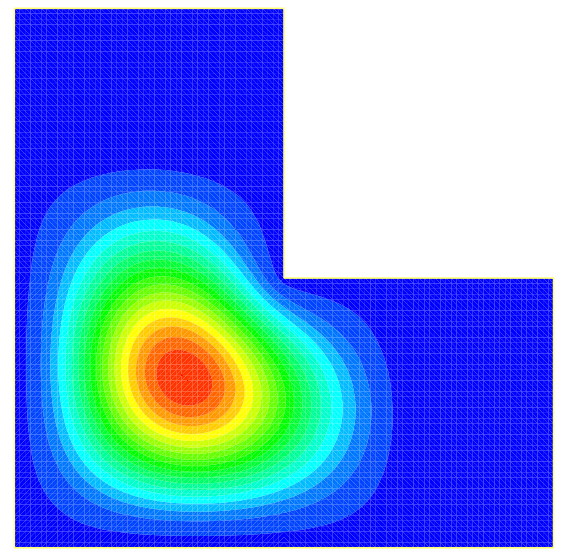

FIGURE 6. Uniformly compressed L-shaped plate; principal buckling mode.

\section{Conclusions}

We have introduced a finite element method for two eigenvalue problems: the computation of buckling and vibration modes of a clamped Kirchhoff polygonal plate. The method is based on discretizing a bending moment formulation by means of standard piecewise linear finite elements. This approach was proposed and analyzed by Amara et al. [1] to solve the corresponding load problem for a thin plate subject to arbitrary boundary conditions.

We have proved that the method yields an $\mathcal{O}(h)$ approximation to the transverse displacements of buckling and vibration modes. Moreover, it yields $\mathcal{O}\left(h^{t}\right)$ approximations to two auxiliary quantities, $\phi$ and $\boldsymbol{\psi}$, which allow us to compute to the same order of accuracy the bending moment $\boldsymbol{\sigma}=\operatorname{Curl} \boldsymbol{\psi}+\frac{1}{2}(\operatorname{div} \boldsymbol{\psi}) \mathbf{J}+\phi \mathbf{I}$. The order $t$ depends on the Sobolev regularity of the domain for the biharmonic and the Laplace equations. If $\Omega$ is convex, then $t=1$; otherwise, $t \in\left(\frac{1}{2}, 1\right)$ depends on the largest re-entrant angle of $\Omega$. The method yields $\mathcal{O}\left(h^{2 t}\right)$ approximation to the buckling coefficients or the vibration frequencies, too.

Furthermore, Lemma 4.4 shows that the method leads to an $\mathcal{O}(h)$ approximation to the transverse displacement in the case of the source problem, too, even for nonconvex polygonal clamped plates. Let us remark that such an optimal order agrees with the fact that the transverse displacement always belongs to $H^{2}(\Omega)$. This improves in this particular case the estimate given in [1, Theorem 5.3] for this variable.

The numerical tests confirm the theoretical results, including the $\mathcal{O}(h)$ approximation to the transverse displacements even for plates with re-entrant corners. The performance of the method analyzed in this paper is comparable to that of the lowest-order Ciarlet-Raviart method [8] (for which, to the best of the authors' knowledge, there is no proof of convergence for either of the eigenvalue problems). 
We have also tested numerically other well-known method for Kirchhoff plates, which was analyzed by Ishihara [13, 14, for both eigenvalue problems on meshes uniform in the interior of the domain. The numerical tests show that the uniformity constraint is not a technicality. In fact, it converges to wrong results when used on particular regular non-uniform meshes.

\section{APPENDIX}

The matrix form of the discrete spectral problem (3.7) reads as follows:

$$
\left(\begin{array}{ccc}
\mathbf{A} & \mathbf{B} & \mathbf{C} \\
\mathbf{B}^{\mathrm{T}} & \mathbf{D} & \mathbf{0} \\
\mathbf{C} & \mathbf{0} & \mathbf{0}
\end{array}\right)\left(\begin{array}{l}
\boldsymbol{\Phi}_{h} \\
\boldsymbol{\Psi}_{h} \\
\mathbf{U}_{h}
\end{array}\right)=\lambda_{h}\left(\begin{array}{ccc}
\mathbf{0} & \mathbf{0} & \mathbf{0} \\
\mathbf{0} & \mathbf{0} & \mathbf{0} \\
\mathbf{0} & \mathbf{0} & -\mathbf{E}
\end{array}\right)\left(\begin{array}{l}
\boldsymbol{\Phi}_{h} \\
\boldsymbol{\Psi}_{h} \\
\mathbf{U}_{h}
\end{array}\right)
$$

where $\boldsymbol{\Phi}_{h}, \mathbf{\Psi}_{h}$, and $\mathbf{U}_{h}$ denote the vectors whose entries are the components of $\phi_{h}, \psi_{h}$, and $u_{h}$, respectively, in given bases of the discrete spaces. Let us remark that $\boldsymbol{\psi}_{h} \in \mathcal{H}_{h}$, whose definition involves three linear constraints. Actually, these constraints are imposed by means of three scalar Lagrange multipliers, which leads to an augmented spectral problem exactly equivalent with (6.1).

In this generalized eigenvalue problem, matrices $\mathbf{A}, \mathbf{C}, \mathbf{D}$, and $\mathbf{E}$ are symmetric, whereas $\mathbf{A}, \mathbf{C}$, and $\mathbf{D}$ are also positive definite. Let us define

$$
\mathbf{F}:=\left(\begin{array}{cc}
\mathbf{A} & \mathbf{B} \\
\mathbf{B}^{\mathrm{T}} & \mathbf{D}
\end{array}\right), \quad \mathbf{G}:=\left(\begin{array}{c}
\mathbf{C} \\
\mathbf{0}
\end{array}\right), \quad \text { and } \quad \mathbf{V}_{h}:=\left(\begin{array}{l}
\mathbf{\Phi}_{h} \\
\boldsymbol{\Psi}_{h}
\end{array}\right)
$$

Matrix $\mathbf{F}$ is non-singular. In fact, the following result holds true:

Lemma 6.1. $\mathbf{F}$ is a positive definite matrix.

Proof. Let $\phi_{h} \in \mathcal{V}_{h}$ and $\boldsymbol{\psi}_{h}=\left(\psi_{h 1}, \psi_{h 2}\right) \in \mathcal{H}_{h}$. Let $\boldsymbol{\Phi}_{h}$ and $\boldsymbol{\Psi}_{h}$ be the vectors whose entries are the components of $\phi_{h}$ and $\boldsymbol{\psi}_{h}$, respectively, and $\mathbf{V}_{h}$ as defined above. Straightforward computations lead to

$$
\begin{aligned}
\mathbf{V}_{h}^{\mathrm{T}} \mathbf{F} \mathbf{V}_{h}= & \frac{2}{1+\nu} \int_{\Omega} \phi_{h}^{2}+\frac{2}{1+\nu} \int_{\Omega}\left(\partial_{2} \psi_{h 1}-\partial_{1} \psi_{h 2}\right) \phi_{h} \\
& +\frac{1}{1-\nu} \int_{\Omega}\left[\left(\partial_{2} \psi_{h 1}\right)^{2}+\left(\partial_{1} \psi_{h 2}\right)^{2}+\frac{1}{2}\left(\partial_{2} \psi_{h 2}-\partial_{1} \psi_{h 1}\right)^{2}\right] \\
& -\frac{\nu}{1-\nu^{2}} \int_{\Omega}\left(\partial_{2} \psi_{h 1}-\partial_{1} \psi_{h 2}\right)^{2} \\
= & \frac{2}{1+\nu} \int_{\Omega}\left[\phi_{h}+\frac{1}{2}\left(\partial_{2} \psi_{h 1}-\partial_{1} \psi_{h 2}\right)\right]^{2} \\
& +\frac{1}{2(1-\nu)} \int_{\Omega}\left[\left(\partial_{2} \psi_{h 1}+\partial_{1} \psi_{h 2}\right)^{2}+\left(\partial_{2} \psi_{h 2}-\partial_{1} \psi_{h 1}\right)^{2}\right] \geq 0 .
\end{aligned}
$$

Hence $\mathbf{F}$ is non-negative definite. Moreover, the expression above vanishes if and only if $\phi_{h}=-\frac{1}{2}\left(\partial_{2} \psi_{h 1}-\partial_{1} \psi_{h 2}\right), \partial_{2} \psi_{h 1}+\partial_{1} \psi_{h 2}=0$ and $\partial_{2} \psi_{h 2}-\partial_{1} \psi_{h 1}=0$. Now, $\phi_{h} \in \mathcal{V}_{h}$ is piecewise linear and continuous, whereas for $\boldsymbol{\psi}_{h} \in \mathcal{H}_{h}, \partial_{2} \psi_{h 1}-$ $\partial_{1} \psi_{h 2}$ is piecewise constant. Hence, if the expression above vanishes, then $\phi_{h}=$ $-\frac{1}{2}\left(\partial_{2} \psi_{h 1}-\partial_{1} \psi_{h 2}\right)$ has to be constant, and, since it vanishes on $\Gamma$, it has to vanish in the whole $\Omega$.

In such a case, $\partial_{2} \psi_{h 1}-\partial_{1} \psi_{h 2}=0$ and $\partial_{2} \psi_{h 1}+\partial_{1} \psi_{h 2}=0$, too, which leads to $\partial_{2} \psi_{h 1}=\partial_{1} \psi_{h 2}=0$. Since $\partial_{2} \psi_{h 2}-\partial_{1} \psi_{h 1}=0$, as well, there holds $\left\|\boldsymbol{\psi}_{h}\right\|_{\mathcal{H}}=0$ and hence $\boldsymbol{\psi}_{h}=\mathbf{0}$. Thus $\mathbf{F}$ is positive definite and we conclude the proof. 
Now we are in a position to prove the following characterization of the discrete spectral problem (3.7):

Proposition 6.2. Let $\mathcal{Z}_{h}:=\left\{u_{h} \in \mathcal{V}_{h}: \mathscr{C}\left(u_{h}, v_{h}\right)=0 \quad \forall v_{h} \in \mathcal{V}_{h}\right\}$. Then, problem (3.7) has exactly $\operatorname{dim} \mathcal{V}_{h}-\operatorname{dim} \mathcal{Z}_{h}$ eigenvalues, repeated according to their respective multiplicities. All of them are real and non-zero.

Proof. Since according to the previous lemma $\mathbf{F}$ is positive definite and consequently non-singular, $\boldsymbol{\Phi}_{h}$ and $\boldsymbol{\Psi}_{h}$ can be eliminated in (6.1) as follows:

$$
\mathbf{V}_{h}=-\mathbf{F}^{-1} \mathbf{G} \mathbf{U}_{h} \quad \Longrightarrow \quad \mathbf{E} \mathbf{U}_{h}=-\mu_{h}\left(\mathbf{G}^{\mathrm{T}} \mathbf{F}^{-1} \mathbf{G}\right) \mathbf{U}_{h},
$$

with $\mu_{h}:=\frac{1}{\lambda_{h}}$ (recall $\lambda_{h} \neq 0$; cf. Remark 3.5).

Now, since also $\mathbf{C}$ is non-singular, the columns of $\mathbf{G}$ are linearly independent. Hence, $\mathbf{G}^{\mathrm{T}} \mathbf{F}^{-1} \mathbf{G}$ is symmetric and positive definite and, $\mathbf{E}$ being symmetric too, the generalized eigenvalue problem $\mathbf{E} \mathbf{U}_{h}=-\mu_{h}\left(\mathbf{G}^{\mathrm{T}} \mathbf{F}^{-1} \mathbf{G}\right) \mathbf{U}_{h}$ is well posed and all its eigenvalues are real. Therefore the number of eigenvalues of problem (6.1) (which is the matrix form of problem (3.7) ) equals the number of non-zero eigenvalues of this problem, namely, $\operatorname{dim} \mathcal{V}_{h}-\operatorname{dim}(\operatorname{Ker}(\mathbf{E}))$. Thus, we conclude the lemma by noting that $\mathbf{E} \mathbf{U}_{h}=\mathbf{0}$ if and only if $u_{h} \in \mathcal{Z}_{h}$.

As an immediate consequence of the proof of this proposition, note that problem (3.7) always has real non-zero eigenvalues, as long as $\mathbf{E} \neq \mathbf{0}$.

Remark 6.3. For all the solutions $\left(\lambda_{h}, \phi_{h}, \psi_{h}, u_{h}\right)$ of problem (3.7), there holds $\int_{\Omega}\left(\boldsymbol{\eta} \nabla u_{h}\right) \cdot \nabla u_{h} \neq 0$, despite the fact that $\boldsymbol{\eta}$ is not necessarily positive definite. In fact, as shown in the proof of Proposition 6.2 that

$$
\int_{\Omega}\left(\boldsymbol{\eta} \nabla u_{h}\right) \cdot \nabla u_{h}=\mathscr{C}\left(u_{h}, u_{h}\right)=\mathbf{U}_{h}^{\mathrm{T}} \mathbf{E} \mathbf{U}_{h}=-\frac{1}{\lambda_{h}} \mathbf{U}_{h}^{\mathrm{T}}\left(\mathbf{G}^{\mathrm{T}} \mathbf{F}^{-1} \mathbf{G}\right) \mathbf{U}_{h} \neq 0 .
$$

\section{ACKNOWLEDGMENT}

The authors thank Ricardo Durán for helpful discussions.

\section{REFERENCES}

1. M. Amara, D. Capatina-Papaghiuc, and A. Chatti, Bending moment mixed method for the Kirchhoff-Love plate model, SIAM J. Numer. Anal., 40 (2002) 1632-1649. MR.1950615 (2003k:74058)

2. M. Amara and F. Dabaghi, An optimal $C^{0}$ finite element algorithm for the $2 D$ biharmonic problem: Theoretical analysis and numerical results, Numer. Math., 90 (2001) 19-46. MR.1868761 (2002h:65172)

3. I. Babuška and J. Osborn, Eigenvalue problems, in Handbook of Numerical Analysis, Vol. II, P.G. Ciarlet and J.L. Lions, eds., North-Holland, Amsterdam, 1991, pp. 641-787. MR1115240

4. I. Babuška, J. Osborn, and J. Pitkäranta, Analysis of mixed methods using mesh dependent norms, Math. Comp., 35 (1980) 1039-1062. MR.583486 (81m:65166)

5. F Brezzi and P.A. Raviart, Mixed finite element methods for 4 th order elliptic equations, in Topics in Numerical Analysis III, J. Miller ed., Academic Press, London, 1977, pp. 33-56. MR0657975 (58:31905)

6. C. Canuto, Eigenvalue approximations by mixed methods, RAIRO Anal. Numér., 12 (1978) 27-50. MR0488712 (58:8229)

7. P.G. Ciarlet, Basic error estimates for elliptic problems, in Handbook of Numerical Analysis, Vol. II, P.G. Ciarlet and J.L. Lions, eds., North Holland, 1991. MR.1115237

8. P.G. Ciarlet and P.-A. Raviart, A mixed finite element method for the biharmonic equation, in Mathematical Aspects of Finite Elements in Partial Differential Equations, C. de Boor, ed., Academic Press, New York, 1974, pp. 125-145. MR0657977 (58:31907) 
9. R. Falk and J. Osborn, Error estimates for mixed methods, RAIRO Anal. Numér., 14 (1980) 249-277. MR.592753 (82j:65076)

10. V. Girault, J. Giroire, and A. Sequeira, A stream function-vorticity variational formulation for the exterior Stokes problem in weighted Sobolev spaces. Math. Meth. Appl. Sci., 15 (1992) 345-363. MR.1170532 (93d:35113)

11. V. Girault and P.A. Raviart, Finite Element Methods for Navier-Stokes Equations, SpringerVerlag, Berlin, 1986. MR851383 (88b:65129)

12. P. Grisvard, Elliptic Problems in Non-Smooth Domains, Pitman, Boston, 1985.

13. K. Ishihara, A mixed finite element method for the biharmonic eigenvalue problem of plate bending, Publ. Res. Inst. Math. Sci., 14 (1978) 399-414. MR509196 (80c:73047)

14. On the mixed finite element approximation for the buckling of plates, Numer. Math., 33 (1979) 195-210. MR549449 (80k:65090)

15. T. Kato, Perturbation Theory for Linear Operators, Springer-Verlag, Berlin, 1995. MR.1335452 (96a:47025)

16. T. Miyoshi, A mixed finite element method for the solution of the von Kármán equations, Numer. Math., 26 (1976) 255-269. MR0438741 (55:11648)

17. B. Mercier, J. Osborn, J. Rappaz, and P. A. Raviart, Eigenvalue approximation by mixed and hybrid methods, Math. Comp., 36 (1981) 427-453. MR606505 (82b:65108)

18. R. Rannacher, Nonconforming finite element methods for eigenvalue problems in linear plate theory, Numer. Math., 33 (1979) 23-42. MR545740 (80i:65124)

19. A. Rössle, Corner singularities and regularity of weak solutions for the two-dimensional Lamé equations on domains with angular corners, J. Elasticity, 60 (2000) 57-75. MR.1832016 (2002c:74030)

20. R. Scholz, A mixed method for 4 th order problems using linear finite elements, RAIRO Anal. Numér., 12 (1978) 85-90. MR0483557|(58:3549)

Departamento de Ingeniería Matemática, Universidad de Concepción, Casilla 160-C, Concepción, Chile

E-mail address: david@ing-mat.udec.cl

Departamento de Ingeniería Matemática, Universidad de Concepción, Casilla 160-C, Concepción, Chile

E-mail address: rodolfo@ing-mat.udec.cl 